\title{
OPEN A generalized strain approach to anisotropic elasticity
}

\author{
M. H. B. M. Shariff
}

This work proposes a generalized Lagrangian strain function $f_{\alpha}$ (that depends on modified stretches) and a volumetric strain function $g_{\alpha}$ (that depends on the determinant of the deformation tensor) to characterize isotropic/anisotropic strain energy functions. With the aid of a spectral approach, the single-variable strain functions enable the development of strain energy functions that are consistent with their infinitesimal counterparts, including the development of a strain energy function for the general anisotropic material that contains the general 4th order classical stiffness tensor. The generality of the single-variable strain functions sets a platform for future development of adequate specific forms of the isotropic/anisotropic strain energy function; future modellers only require to construct specific forms of the functions $f_{\alpha}$ and $g_{\alpha}$ to model their strain energy functions. The spectral invariants used in the constitutive equation have a clear physical interpretation, which is attractive, in aiding experiment design and the construction of specific forms of the strain energy. Some previous strain energy functions that appeared in the literature can be considered as special cases of the proposed generalized strain energy function. The resulting constitutive equations can be easily converted, to allow the mechanical influence of compressed fibres to be excluded or partial excluded and to model fibre dispersion in collagenous soft tissues. Implementation of the constitutive equations in Finite Element software is discussed. The suggested crude specific strain function forms are able to fit the theory well with experimental data and managed to predict several sets of experimental data.

Hill ${ }^{1}$ introduced a generalized strain function in finite elasticity. Using a Hill's strain function of the form

$$
\frac{\lambda^{\kappa}-1}{\kappa}
$$

where $\kappa$ is real parameter and $\lambda$ is a principal stretch, Ogden ${ }^{2}$ successfully model the mechanical behaviour of incompressible isotropic solids. In the literature, different values of the parameter $\kappa$ are used to model different types of incompressible isotropic elastic solids; this suggests that the selection of strain functions to model a constitutive equation depends on the type of material we intend to model. Several special forms of the Hill's generalized strain function used in modelling anisotropic/isotropic elastic solids can be found, for example in references $^{3-7}$. In general, Hill's strain invariants do not depend explicitly on right Cauchy-Green tensor $\boldsymbol{C}$ and their 1st and 2nd order derivatives with respect to $C$ can only be obtained via spectral derivative formulae that are recently developed (see, for example references ${ }^{8-11}$ ) and, in view of this, the author believes that anisotropic/ isotropic strain energy functions that are characterized by Hill's generalized strain functions (to the best of the author's knowledge) do not exist in the literature. This motivates the author to develop infinitesimal-consistent anisotropic/isotropic finite strain energy functions that are based on the generalized Hill's strain function and the development requires proposing modified Hill's and volumetric strain functions; it also requires a spectral approach based on the author's previous work on anisotropic spectral models (see, for example references ${ }^{12-15}$ ) that used spectral invariants with a clear physical meaning. The advantages of spectral invariants over classical invariants ${ }^{16}$ in constitutive modelling are described, for example, in Shariff and Merodio ${ }^{17}$, hence we will not elaborate them here. In the excellent work of references ${ }^{18-22}$, spectral invariants have also been used to construct an infinitesimal-consistent anisotropic/isotropic finite strain energy function via the WYPiWYG approach, where the energy function shape and the material data of the model are obtained solving the equilibrium equations of the different experiments. In future, there might be a possibility to connect our approach to the WYPiWYG approach. Using an approach similar to that given in references ${ }^{8,9,15,23}$, our proposed model may be extended to model dissipative materials such as those discussed $\mathrm{in}^{24-26}$. Our proposed model may also be possibly extended to model strain gradient materials (see, for example references ${ }^{26,27}$ ) via a similar approach to that of Soltadtos et al. ${ }^{28}$. 
The number of independent spectral invariants in an irreducible/minimal integrity basis can be easily derived ${ }^{29-32}$. A classical irreducible/minimal integrity basis, for a highly anisotropic material, contains a numerous amount of classical invariants ${ }^{16}$ and, due to their unclear physical interpretation, it is not clear in the literature how to select an appropriate (or optimum) subset of classical invariants from an irreducible/minimal integrity basis to represent a strain energy function: In particular, most traditional invariant-based approach to hyperelasticity, which uses classical invariants, typically allows discretion to deem which invariants are necessary for inclusion in the strain energy function for a given model. Omission of invariants offers mathematically simplified models and reduced number of ground-state material constants required for calibration (Shariff ${ }^{12,14}$ has shown that some of the well known models in the literature do not contain all their ground-state constants). However, the discrimination in selection of invariants is often debated, and neglecting the influence of some invariants may result in an incomplete representation of the full range of mechanical response subjected to a continuum ${ }^{14,33-35}$. In this communication, we show that the construction of a strain energy function that uses a full set of spectral invariants that is consistent with infinitesimal theory can be easily done via the use of, modified Hill's and volumetric strain functions: A discussion on the importance of a nonlinear (finite strain) strain energy function that must be consistent with infinitesimal theory can be found, for example in, Rosa et al. ${ }^{22}$ and Shariff ${ }^{14}$.

In some materials, the mechanical influence of compressed fibres is negligible or is different from stretched fibres and, in soft tissues, the influence of fibre dispersion could be relevant in modelling constitutive equations: In Appendices A and B (Supplementary information), we illustrate how the proposed strain energy functions can be easily amended to take account of these influences.

Remark. Valanis and Landel ${ }^{36}$ strain energy function

$$
W_{(v)}=\sum_{i=1}^{3} \bar{r}\left(\lambda_{i}\right),
$$

where $\lambda_{i}$ s are principal stretches, play an important role in modelling incompressible isotropic solids. The function $\bar{r}$ is arbitrary and this set a platform for modelling specific types of incompressibe isotropic elastic solids. Numerous specific forms of $\bar{r}$, that are able to successfully model the mechanical behaviour of incompressible isotropic solids, have been proposed in the literature, see for example Ogden ${ }^{2}$ and Shariff ${ }^{37}$. The single variable function $\bar{r}$ depends on an invariant with a clear physical meaning and this makes the Valanis and Landel form experimentally attractive ${ }^{17}$. The Valanis and Landel form impels us to develop anisotropic constitutive equations, which depends on single variable aribtrary functions that will set a platform for future modelling of specific types of anisotropic elastic solids. Our constitutive equations are developed via generalized strain single-variable functions. We overtly emphasize that, in this paper, we are not particularly concerned in obtaining specific forms of the proposed generalized strain functions. A rigourous construction of specific forms such those found in references ${ }^{2,14,37}$ requires a lot of work and it is beyond the scope of this paper. As mentioned above, the generalized constitutive equations described here will set a platform (analogous to the "generalized" Valanis and Landel form for isotropic elastic solids) for future modelling of specific types of anisotropic elastic solids.

\section{Preliminaries}

In this paper, summation convention is not used and all subscripts $i, j$ and $k$ take the values $1,2,3$, unless stated otherwise. Vectors and tensors are written in lowercase and uppercase bold fonts, respectively. Only quasistatic deformations and time-independent fields are considered. The mechanical body forces are assumed to be negligible. The deformation gradient is denoted by $\boldsymbol{F}$ and $\boldsymbol{C}=\boldsymbol{F}^{T} \boldsymbol{F}=\boldsymbol{U}^{2}$, respectively, where $\boldsymbol{U}$ is the right stretch tensor.

\section{General strain energy function}

A general strain energy function for an elastic solid can be expressed as

$$
W_{(A)}=W_{a}(\boldsymbol{U}) .
$$

The facilitate the construction of an incompressible material, regarded as a material recovered from the corresponding compressible material by taking the incompressible limit ${ }^{38}$, we use the modified stretch tensor

$$
\boldsymbol{U}^{*}=J^{-\frac{1}{3}} \boldsymbol{U}, \quad \operatorname{det} \boldsymbol{U}^{*}=1,
$$

where $J=\operatorname{det} \boldsymbol{F}>0$ and det is the determinant of a tensor. Hence, we express

$$
W_{(A)}=W_{a}(\boldsymbol{U})=W^{*}\left(\boldsymbol{U}^{*}, J\right) .
$$

The spectral representation

$$
\boldsymbol{U}^{*}=\sum_{i=1}^{3} \lambda_{i}^{*} \boldsymbol{u}_{i} \otimes \boldsymbol{u}_{i},
$$

where $\lambda_{i}^{*}=J^{-\frac{1}{3}} \lambda_{i}, \otimes$ denotes the dyadic product and, $\lambda_{i}$ and $\boldsymbol{u}_{i}$ are an eigenvalue and an orthonormal eigenvector of $\boldsymbol{U}$, respectively. In view of (6),

$$
W_{(A)}=W^{*}\left(\boldsymbol{U}^{*}, J\right)=W\left(\lambda_{1}^{*}, \lambda_{2}^{*}, \lambda_{3}^{*}, \boldsymbol{u}_{1}, \boldsymbol{u}_{2}, \boldsymbol{u}_{2}, J\right) .
$$


Since $\lambda_{1}^{*} \lambda_{2}^{*} \lambda_{3}^{*}=1$ only 6 of the 7 arguments in (7) are independent. The strain energy function $W$ must satisfy the $P$-property described in ${ }^{13}$, that is associated with the symmetrical property of $W$ and the unique value of $W$ when two or more of the principal axes has the same value.

Stress. The Cauchy stress for a compressible solid is

$$
\boldsymbol{T}=\frac{2}{J} \boldsymbol{F} \frac{\partial W_{(A)}}{\partial \boldsymbol{C}} \boldsymbol{F}^{T}
$$

and for an incompressible solid

$$
\boldsymbol{T}=2 \boldsymbol{F} \frac{\partial W_{(A)}}{\partial \boldsymbol{C}} \boldsymbol{F}^{T}-p \boldsymbol{I},
$$

where $p$ is the Lagrange multiplier associated with the constraint $\operatorname{det} \boldsymbol{F}=1$ and $\boldsymbol{I}$ is the identity tensor. Following the work of ${ }^{10,17}$, the Cauchy stress $\boldsymbol{T}$ with respect to the Eulerian orthonormal basis $\left\{\boldsymbol{v}_{1}, \boldsymbol{v}_{2}, \boldsymbol{v}_{3}\right\}$, where $\boldsymbol{v}_{i}=\boldsymbol{R} \boldsymbol{u}_{i}$ and $\boldsymbol{R}=\boldsymbol{F} \boldsymbol{U}^{-1}$ takes the form

$$
\boldsymbol{T}=\sum_{i, j=1}^{3} t_{i j} \boldsymbol{v}_{i} \otimes \boldsymbol{v}_{j}, \quad t_{i j}=\boldsymbol{v}_{i} \cdot \boldsymbol{T} \boldsymbol{v}_{j}
$$

where

$$
\begin{aligned}
J t_{i i}=\lambda_{i}^{*} \frac{\partial W}{\partial \lambda_{i}^{*}}-p^{*}, \quad J t_{i j} & =\frac{\lambda_{i}^{*} \lambda_{j}^{*}}{\lambda_{i}^{* 2}-\lambda_{j}^{* 2}}\left(\frac{\partial W}{\partial \boldsymbol{u}_{i}} \cdot \boldsymbol{u}_{j}-\frac{\partial W}{\partial \boldsymbol{u}_{j}} \cdot \boldsymbol{u}_{i}\right), \quad i \neq j, \\
p^{*} & =\frac{1}{3} \sum_{i=1}^{3} \lambda_{i}^{*} \frac{\partial W}{\partial \lambda_{i}^{*}}-J \frac{\partial W}{\partial J},
\end{aligned}
$$

for compressible elastic solids and in the case for an incompressible solid $(J=1)$, we have,

$$
t_{i i}=\lambda_{i} \frac{\partial W}{\partial \lambda_{i}}-p, \quad t_{i j}=\frac{\lambda_{i} \lambda_{j}}{\lambda_{i}^{2}-\lambda_{j}^{2}}\left(\frac{\partial W}{\partial \boldsymbol{u}_{i}} \cdot \boldsymbol{u}_{j}-\frac{\partial W}{\partial \boldsymbol{u}_{j}} \cdot \boldsymbol{u}_{i}\right), \quad i \neq j .
$$

The nominal stress

$$
\boldsymbol{S}=J \boldsymbol{F}^{-1} \boldsymbol{T}=\sum_{i, j=1}^{3} \frac{J t_{i j}}{\lambda_{i}} \boldsymbol{u}_{i} \otimes \boldsymbol{v}_{j}
$$

It is clear from the above that hydrostatic stress for a compressible material

$$
\frac{\operatorname{tr} \boldsymbol{T}}{3}=\frac{1}{3} \sum_{i=1}^{3} t_{i i}=\frac{\partial W}{\partial J}\left(\lambda_{1}^{*}, \lambda_{2}^{*}, \lambda_{3}^{*}, \boldsymbol{u}_{1}, \boldsymbol{u}_{2}, \boldsymbol{u}_{2}, J\right) .
$$

In the incompressibility limit, the value of $\lim _{J \rightarrow 1} \frac{\partial W}{\partial J}$ and the appropriate properties of $W$ are discussed in Shariff and Parker ${ }^{38}$. We note that all the proposed strain energy functions in this paper are consistent with the strain energy functions proposed by Shariff and Parker $^{38}$. The deformation dependent bulk modulus is defined as ${ }^{39}$

$$
B(\boldsymbol{U})=\frac{\partial^{2} W}{\partial J^{2}}\left(\lambda_{1}^{*}, \lambda_{2}^{*}, \lambda_{3}^{*}, \boldsymbol{u}_{1}, \boldsymbol{u}_{2}, \boldsymbol{u}_{2}, J\right)
$$

The ground-state bulk modulus is defined as

$$
\chi=B(I)
$$

\section{Generalized strain}

Consider a set of general class of Lagrangean strain tensor $\boldsymbol{F}_{(\alpha)}$, similar to that defined by Hill ${ }^{1}$,

$$
\boldsymbol{F}_{(\alpha)}(\boldsymbol{U})=\sum_{i=1}^{3} f_{\alpha}\left(\lambda_{i}^{*}\right) \boldsymbol{u}_{i} \otimes \boldsymbol{u}_{i}, \quad \alpha \in N
$$

where $N=\{1,2,3, \ldots\}$ is the set of natural numbers excluding 0 and $f_{\alpha}:(0, \infty) \rightarrow \mathbb{R}$ is a monotonic increasing function, i.e, $f_{\alpha}^{\prime}\left(\lambda_{i}^{*}\right)>0$, such that

$$
f_{\alpha}(1)=0, \quad f_{\alpha}^{\prime}(1)=1
$$


We could also include, when appropriate, $f_{\alpha}$ to represent physical strain measures with the extreme deformation values

$$
f_{\alpha}\left(\lambda_{i}^{*} \rightarrow \infty\right)=\infty, \quad f_{\alpha}\left(\lambda_{i}^{*} \rightarrow 0\right)=-\infty .
$$

An example of a strain measure commonly used in the literature that satisfies the above properties is

$$
\ln \left(\boldsymbol{U}^{*}\right)=\sum_{i=1}^{3} \ln \left(\lambda_{i}^{*}\right) \boldsymbol{u}_{i} \otimes \boldsymbol{u}_{i}, \quad f_{\alpha}(x)=\ln x .
$$

A strain function that could be of interest, which is similar to the Ogden's strain function ${ }^{2}$, is

$$
f_{\alpha}(x)=\frac{1}{m} \sum_{n=1}^{m} \frac{x^{\bar{\alpha}_{n}}-1}{\bar{\alpha}_{n}}, \quad x>0,
$$

where $\bar{\alpha}$ is a real number. We strongly emphasize that we are not concerned with proposing prototypes of the strain function $f_{\alpha}$, such as those expressed in (21) and (22). An objective of this paper is to construct constitutive equations that depend explicitly on the arbitrary functions $f_{\alpha}$ and $g_{\alpha}$ (defined below), and are consistent with infinitesimal elasticity.

We define a volumetric strain

$$
g_{\alpha}(J), \quad \alpha \in N
$$

where $g_{\alpha}:(0, \infty) \rightarrow \mathbb{R}$ is a monotonic increasing function such that

$$
g_{\alpha}(1)=0, \quad g_{\alpha}^{\prime}(1)=1
$$

We also include, if required, $g_{\alpha}$ to represent physical volumetric strain measures with the extreme deformation values

$$
g_{\alpha}(J \rightarrow \infty)=\infty, \quad g_{\alpha}(J \rightarrow 0)=-\infty .
$$

An example of a volumetric strain is

$$
g_{1}(J)=\ln J
$$

Note that in view of (18), we have, for example

$$
\operatorname{tr}\left(\boldsymbol{F}_{(\alpha)} \boldsymbol{F}_{(\beta)}\right)=\sum_{i=1}^{3} f_{\alpha}\left(\lambda_{i}^{*}\right) f_{\beta}\left(\lambda_{i}^{*}\right), \quad \alpha, \beta \in N .
$$

It is clear from the properties of $f_{\alpha}$ that

$$
f_{\alpha}\left(\lambda_{i}^{*}\right) f_{\beta}\left(\lambda_{i}^{*}\right) \geq 0
$$

Infinitesimal strain. In infinitesimal strain

$$
|\boldsymbol{F}-\boldsymbol{I}|=\left|\frac{\partial \boldsymbol{u}}{\partial \boldsymbol{x}}\right|=O(e),
$$

where $\boldsymbol{u}$ is the displacement vector, $|\bullet|$ is an appropriate norm and the magnitude of $e$ is much less than unity. Up to $O(e)$, we have

$$
\boldsymbol{U}-\boldsymbol{I} \approx \boldsymbol{E}, \quad \boldsymbol{U}^{*}-\boldsymbol{I} \approx \boldsymbol{E}^{*}, \quad f_{\alpha}\left(\lambda_{i}\right) \approx \lambda_{i}-1=e_{i}, \quad f_{\alpha}\left(\lambda_{i}^{*}\right) \approx \lambda_{i}^{*}-1=e_{i}^{*},
$$

where $e_{i}$ is an eigenvalue of the infinitesimal strain $\boldsymbol{E}$ (we do not distinguish the eigenvectors of $\boldsymbol{U}$ and $\boldsymbol{E}$ ) and $e_{i}^{*}$ is an eigenvalue of

$$
\boldsymbol{E}^{*}=\boldsymbol{E}-\frac{1}{3}(\operatorname{tr} \boldsymbol{E}) \boldsymbol{I}
$$

Spectrally, we can express

$$
\boldsymbol{E}=\sum_{i=1}^{3} e_{i} \boldsymbol{u}_{i} \otimes \boldsymbol{u}_{i}, \quad \boldsymbol{E}^{*}=\sum_{i=1}^{3} e_{i}^{*} \boldsymbol{u}_{i} \otimes \boldsymbol{u}_{i},
$$

where the eigenvalues

$$
e_{i}^{*}=e_{i}-\frac{e_{1}+e_{2}+e_{3}}{3} .
$$

Up to order $O(e)$, the volumetric strain 


$$
g_{\alpha}(J) \approx J-1=\operatorname{tr} \boldsymbol{E} .
$$

Remark. In this paper, the construction of a strain energy function for finite strain deformations that is consistent with infinitesimal elasticity is facilitated via infinitesimal strain elasticity. Hence, in sections "Isotropic" to "General anisotropy", we start the construction of a finite strain constitutive equation with the development of its infinitesimal strain energy function counterpart.

For the sake of generality, the general constitutive equations given below contain numerous functions of $f_{\alpha}$ and $g_{\alpha}$, which may seem unappealing. However, in many occasions only a few $f_{\alpha}$ and $g_{\alpha}$ functions are required to model anisotropic solids (see "Example of specific forms of $f \alpha$ and $g \alpha$ used in experimental fitting" section).

\section{Isotropic}

Let $W_{(I)}$ represents the strain energy for an isotropic elastic solid. We then have

$$
W_{(I)}=W_{I}(\boldsymbol{U})=W_{I}\left(\boldsymbol{Q U} \boldsymbol{Q} \boldsymbol{Q}^{T}\right)
$$

where $\boldsymbol{Q}$ is an arbitrary rotational tensor and (35) implies that the strain energy $W_{(I)}$ can be symmetrically express in terms the principal stretches (spectral invariants) $\lambda_{i}$.

Infinitesimal strain. The strain energy function for infinitesimal strain deformations is

$$
W_{(I)}=\mu \operatorname{tr} \boldsymbol{E}^{2}+\frac{\lambda}{2}(\operatorname{tr} \boldsymbol{E})^{2}=\mu \operatorname{tr} \boldsymbol{E}^{* 2}+\frac{\chi}{2}(\operatorname{tr} \boldsymbol{E})^{2},
$$

where $\mu$ and $\chi$ are, respectively, the ground state shear and bulk moduli and $\lambda$ is the Lame's constant. For the purpose of this paper, we express

$$
W_{(I)}=\mu \sum_{i=1} e_{i}^{* 2}+\frac{\chi}{2} h^{2}
$$

where $h=\operatorname{tr} E$. In the case of an incompressible solid, (37) is reduced to

$$
W_{(I)}=\mu \sum_{i=1} e_{i}^{2}
$$

Finite strain. A finite strain energy function that is consistent with its infinitesimal counterpart (37) is proposed, i.e.,

$$
W_{(I)}=\mu \operatorname{tr}\left(\boldsymbol{F}_{(1)} \boldsymbol{F}_{(2)}\right)+\frac{\chi}{2} g_{1}(J) g_{2}(J)+\phi_{(I)}=\mu \sum_{i=1}^{3} f_{1}\left(\lambda_{i}^{*}\right) f_{2}\left(\lambda_{i}^{*}\right)+\frac{\chi}{2} g_{1}(J) g_{2}(J)+\phi_{(I)},
$$

where the "higher order" term $\phi_{(I)}$ (which depends on $\lambda_{i}$ ) satisfies the $P$-property and the conditions

$$
\phi_{(I)}=0, \quad \frac{\partial \phi_{(I)}}{\partial \boldsymbol{U}}=\mathbf{0}, \quad \frac{\partial^{2} \phi_{(I)}}{\partial \boldsymbol{U} \partial \boldsymbol{U}}=\mathbf{0}
$$

at $\boldsymbol{F}=\boldsymbol{I}$. We note that, in view of (40), the function $\phi_{(I)}$ does not contribute to the infinitesimal strain energy function. In the case of an incompressible material, we propose

$$
W_{(I)}=\mu \sum_{i=1}^{3} f_{1}\left(\lambda_{i}\right) f_{2}\left(\lambda_{i}\right)+\phi_{(I)}
$$

For neatness, we have used the same expression for $\phi_{(I)}$ in (39) and (41) although they are, generally, different functions.

The weighted Cauchy stress takes the form

$$
J \boldsymbol{T}=\sum_{i=1}^{3}\left(\lambda_{i}^{*} \frac{\partial W_{(I)}}{\partial \lambda_{i}^{*}}-p^{*}\right) \boldsymbol{v}_{i} \otimes \boldsymbol{v}_{i}
$$

Examples of strain energy functions in the literature that can be written in the forms (39) and (41) are given below:

(a) For compressible materials with $\Phi_{(I)}=0$ :

1. The Hencky ${ }^{40}$ strain energy function

$$
W_{(I)}=\mu \sum_{i=1}^{3}\left(\ln \left(\lambda_{i}^{*}\right)\right)^{2}+\frac{\chi}{2}(\ln J)^{2}=\mu \sum_{i=1}^{3}\left(\ln \left(\lambda_{i}\right)\right)^{2}+\frac{\lambda}{2}(\ln J)^{2} .
$$


In this case, we have,

$$
f_{1}\left(\lambda_{i}^{*}\right)=f_{2}\left(\lambda_{i}^{*}\right)=\ln \left(\lambda_{i}^{*}\right), \quad g_{1}(J)=g_{2}(J)=\ln J .
$$

2. A Mooney-Rivlin strain energy function

$$
\begin{aligned}
W_{(I)} & =c_{1}\left(\sum_{i=1}^{3} \lambda_{i}^{* 2}-3\right)+c_{2}\left(\sum_{i=1}^{3} \frac{1}{\lambda_{i}^{* 2}}-3\right)+\frac{\chi}{2}(J-1)^{2} \\
& =c_{1} \sum_{i=1}^{3}\left(\lambda_{i}^{* 2}-2 \ln \lambda_{i}^{*}-1\right)+c_{2} \sum_{i=1}^{3}\left(\frac{1}{\lambda_{i}^{* 2}}+2 \ln \lambda_{i}^{*}-1\right)+\frac{\chi}{2}(J-1)^{2} .
\end{aligned}
$$

In this case, we have,

$$
\begin{aligned}
\mu & =2\left(c_{1}+c_{2}\right), \quad f_{1}\left(\lambda_{i}^{*}\right)=f_{2}\left(\lambda_{i}^{*}\right)=f\left(\lambda_{i}^{*}\right), \\
f\left(\lambda_{i}^{*}\right)^{2} & =\frac{1}{\mu}\left(c_{1}\left(\lambda_{i}^{* 2}-2 \ln \lambda_{i}^{*}-1\right)+c_{2}\left(\frac{1}{\lambda_{i}^{* 2}}+2 \ln \lambda_{i}^{*}-1\right)\right) \geq 0, \\
g_{1}(J) & =g_{2}(J)=(J-1),
\end{aligned}
$$

for appropriate values of $c_{1}$ and $c_{2}$. Note that, we can also compare our model with Mooney-Rivlin strain energy function, where $f_{1} \neq f_{2}$. For example,

$$
\begin{aligned}
& f_{1}\left(\lambda_{i}^{*}\right)=\left(\lambda_{i}^{*}-1\right), \\
& f_{2}\left(\lambda_{i}^{*}\right)=\frac{1}{\mu\left(\lambda_{i}^{*}-1\right)}\left(c_{1}\left(\lambda_{i}^{* 2}-2 \ln \lambda_{i}^{*}-1\right)+c_{2}\left(\frac{1}{\lambda_{i}^{* 2}}+2 \ln \lambda_{i}^{*}-1\right)\right),
\end{aligned}
$$

taking note that

$$
\lim _{\lambda_{i}^{*} \rightarrow 1} f_{2}\left(\lambda_{i}^{*}\right)=0, \quad \lim _{\lambda_{i}^{*} \rightarrow 1} f_{2}^{\prime}\left(\lambda_{i}^{*}\right)=1 .
$$

(b) Incompressible materials with $\Phi_{(I)}=0$ :

1. The Valanis and Landel ${ }^{36}$ form

$$
W_{(I)}=\sum_{i=1}^{3} r\left(\lambda_{i}\right)
$$

In this case

$$
f_{1}\left(\lambda_{i}\right)=f_{2}\left(\lambda_{i}\right)=f\left(\lambda_{i}\right), \quad f\left(\lambda_{i}\right)^{2}=\frac{1}{\mu} r\left(\lambda_{i}\right),
$$

with the conditions

$$
r(1)=r^{\prime}(1)=0, \quad r^{\prime \prime}(1)=2 \mu .
$$

2. Ogden $^{2}$ strain energy function

$$
W_{(I)}=\sum_{r} \frac{\mu_{r}}{\alpha_{r}}\left(\lambda_{1}^{\alpha_{r}}+\lambda_{2}^{\alpha_{r}}+\lambda_{3}^{\alpha_{r}}-3\right) .
$$

In this case, we have,

$$
\begin{aligned}
\mu & =\frac{1}{2} \sum_{r} \mu_{r} \alpha_{r}, \quad f_{1}\left(\lambda_{i}\right)=f_{2}\left(\lambda_{i}\right)=f\left(\lambda_{i}\right), \\
f\left(\lambda_{i}\right)^{2} & =\frac{1}{\mu} \sum_{r} \frac{\mu_{r}}{\alpha_{r}}\left(\lambda_{i}^{\alpha_{r}}-\alpha_{r} \ln \left(\lambda_{i}\right)-1\right) \geq 0,
\end{aligned}
$$

for appropriate values of the material constants $\mu_{r}$ and $\alpha_{r}$. It is worth noting that

$$
\lambda_{i}^{\alpha_{r}}-\alpha_{r} \ln \left(\lambda_{i}\right)-1 \geq 0 .
$$

Remark: In sections "Transversely Isotropic with a unit preferred direction $a$ " and "Two preferred direction elastic solid" below, we discuss elastic solids with one and two preferred directions. In some of these solids, the mechanical influence of compressed fibres is negligible or is different from stretched fibres and in some soft tissue solids, the influence of fibre dispersion could be relevant in modelling constitutive equations: In Appendices A and B (Supplementary information), we illustrate how the strain energy functions developed in sections "Transversely Isotropic with a unit preferred direction $a$ " and "Two preferred direction elastic solid" can be easily amended to take account of these influences. 
Transversely Isotropic with a unit preferred direction $a$

Let $W_{(T)}$ represents the strain energy for a transversely isotropic elastic solid. We then have

$$
W_{(T)}=\bar{W}_{T}(\boldsymbol{U}, \boldsymbol{a} \otimes \boldsymbol{a})=W_{T}(\boldsymbol{U}, \boldsymbol{a})=W_{T}\left(\boldsymbol{Q U} \boldsymbol{Q}^{T}, \boldsymbol{Q a}\right) .
$$

Following the work of Shariff ${ }^{14}$, we can express the strain energy function in terms of the spectral invariants

$$
\lambda_{i}, \quad a_{i}=\boldsymbol{u}_{i} \cdot \boldsymbol{a}=\boldsymbol{Q} \boldsymbol{u}_{i} \cdot \boldsymbol{Q} \boldsymbol{a} .
$$

Since, $\boldsymbol{a}$ is a unit vector, we have,

$$
\sum_{i=1}^{3} \zeta_{i}=1, \quad \zeta_{i}=a_{i}^{2}
$$

and hence only 5 of the 6 invariants in (56) are independent ${ }^{31,32}$.

Infinitesimal strain. The infinitesimal strain energy function is ${ }^{41}$

$$
W_{(T)}=\bar{a}_{1} \operatorname{tr} \boldsymbol{E}^{2}+\bar{a}_{2}(\operatorname{tr} \boldsymbol{E})^{2}+\bar{a}_{3} \operatorname{tr}\left(\boldsymbol{A} \boldsymbol{E}^{2}\right)+\bar{a}_{4}(\operatorname{tr}(\boldsymbol{A E}))^{2}+\bar{a}_{5} \operatorname{tr}(\boldsymbol{A E}) \operatorname{tr} \boldsymbol{E},
$$

where $\boldsymbol{A}=\boldsymbol{a} \otimes \boldsymbol{a}$. The material constants $\bar{a}_{i}(i=1,2, \ldots, 5)$ can be be described in terms of physical parameters as shown below:

$$
\begin{aligned}
& \bar{a}_{1}=\frac{a_{11}-a_{12}}{2}, \quad \bar{a}_{2}=\frac{a_{12}}{2}, \quad \bar{a}_{3}=a_{44}-a_{11}+a_{12}, \quad \bar{a}_{4}=\frac{a_{33}}{2}+\frac{a_{11}}{2}-a_{44}-a_{13}, \\
& \bar{a}_{5}=a_{13}-a_{12},
\end{aligned}
$$

where

$$
\begin{gathered}
a_{11}=\frac{1-v_{a} v_{z p}}{E_{p} E_{a} D}, \quad a_{12}=\frac{v_{p}+v_{z p} v_{a}}{E_{p} E_{a} D}, \quad a_{13}=\frac{v_{a}\left(1+v_{p}\right)}{E_{p}^{2} D}, \quad a_{33}=\frac{1-v_{p}^{2}}{E_{p}^{2} D}, \quad a_{44}=2 \mu_{a}, \\
D=\frac{\left(1+v_{p}\right)\left(1-v_{p}-2 v_{a} v_{z p}\right)}{E_{p}^{2} E_{a}} .
\end{gathered}
$$

Here $v_{p}$ is the Poisson ratio in a particular direction on the plane of symmetry, when the material is extended in a direction on the plane of symmetry perpendicular to the particular direction, $v_{a}$ is the Poisson ratio in the preferred direction when the material is extended in the plane of symmetry, $E_{p}$ is the Young's Modulus in the plane of symmetry normal to the preferred direction $\boldsymbol{a}, \mu_{a}$ is the shear modulus in the preferred direction and $E_{a}$ is the Young's modulus in the preferred direction. Take note that we have also the relation

$$
\frac{v_{a}}{E_{p}}=\frac{v_{z p}}{E_{a}}
$$

where $v_{z p}$ is the Poisson ratio in any direction on the plane of symmetry, when the material is extended in the preferred direction.

We can express (58) in the form

$$
W_{(T)}=W_{T}\left(\boldsymbol{E}^{*}, h\right)=\bar{a}_{1} \operatorname{tr} \boldsymbol{E}^{* 2}+a_{(2)} h^{2}+\bar{a}_{3} \boldsymbol{a} \cdot \boldsymbol{E}^{* 2} \boldsymbol{a}+\bar{a}_{4}\left(\boldsymbol{a} \cdot \boldsymbol{E}^{*} \boldsymbol{a}\right)^{2}+a_{(5)} h \boldsymbol{a} \cdot \boldsymbol{E}^{*} \boldsymbol{a},
$$

where

$$
a_{(2)}=\frac{\bar{a}_{1}}{3}+\bar{a}_{2}+\frac{\bar{a}_{3}}{9}+\frac{\bar{a}_{4}}{9}+\frac{\bar{a}_{5}}{3}, \quad a_{(5)}=\frac{2 \bar{a}_{3}}{3}+\frac{2 \bar{a}_{4}}{3}+\bar{a}_{5} .
$$

The infinitesimal hydrostatic stress

$$
\frac{\operatorname{tr} \boldsymbol{T}}{3}=\frac{\partial W_{T}}{\partial h}\left(\boldsymbol{E}^{*}, h\right)=2 a_{(2)} h+a_{(5)} \boldsymbol{a} \cdot \boldsymbol{E}^{*} \boldsymbol{a} .
$$

The ground-state bulk modulus then takes the form

$$
\chi=\frac{\partial^{2} W_{T}}{\partial h^{2}}\left(\boldsymbol{E}^{*}, h\right)=2 a_{(2)} .
$$

It can be easily shown that, in the incompressible limit, as $v_{z p} \rightarrow 0.5$ and $1-v_{a}-v_{p} \rightarrow 0^{42}$ the ground-state bulk modulus $\chi \rightarrow \infty$. It is clear from (65) that, since,

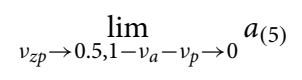

exists, we have 


$$
h=\operatorname{tr} E \rightarrow 0 \quad \text { as } \quad \chi \rightarrow \infty
$$

In spectral form,

$$
\operatorname{tr} \boldsymbol{E}^{* 2}=\sum_{i=1}^{3} e_{i}^{* 2}, \quad \boldsymbol{a} \cdot \boldsymbol{E}^{*} \boldsymbol{a}=\sum_{i=1}^{3} \zeta_{i} e_{i}^{*}, \quad \boldsymbol{a} \cdot \boldsymbol{E}^{* 2} \boldsymbol{a}=\sum_{i=1}^{3} \zeta_{i} e_{i}^{* 2}
$$

Finite strain. Using (63) and (69), we easily construct a finite strain energy that it is consistent with its infinitesimal counterpart, i.e.

$$
\begin{aligned}
W_{(T)}= & \bar{a}_{1} \operatorname{tr}\left(\boldsymbol{F}_{(1)} \boldsymbol{F}_{(2)}\right)+a_{(2)} g_{1}(J) g_{2}(J)+\bar{a}_{3} \boldsymbol{a} \cdot \boldsymbol{F}_{(3)} \boldsymbol{F}_{(4)} \boldsymbol{a} \\
& +\bar{a}_{4}\left(\boldsymbol{a} \cdot \boldsymbol{F}_{(5)} \boldsymbol{a}\right)\left(\boldsymbol{a} \cdot \boldsymbol{F}_{(6)} \boldsymbol{a}\right)+a_{(5)} g_{3}(J) \boldsymbol{a} \cdot \boldsymbol{F}_{(7)} \boldsymbol{a}+\phi_{(I)}
\end{aligned}
$$

or, alternatively,

$$
\begin{aligned}
W_{(T)}= & \bar{a}_{1} \sum_{i=1}^{3} f_{1}\left(\lambda_{i}^{*}\right) f_{2}\left(\lambda_{i}^{*}\right)+a_{(2)} g_{1}(J) g_{2}(J)+\bar{a}_{3} \sum_{i=1}^{3} \zeta_{i} f_{3}\left(\lambda_{i}^{*}\right) f_{4}\left(\lambda_{i}^{*}\right) \\
& +\bar{a}_{4}\left(\sum_{i=1}^{3} \zeta_{i} f_{5}\left(\lambda_{i}^{*}\right)\right)\left(\sum_{i=1}^{3} \zeta_{i} f_{6}\left(\lambda_{i}^{*}\right)\right)+a_{(5)} g_{3}(J) \sum_{i=1}^{3} \zeta_{i} f_{7}\left(\lambda_{i}^{*}\right)+\phi_{(I)},
\end{aligned}
$$

where the higher order function $\phi_{(I)}$ (for convenient, we use the same expression for all anisotropic material discussed in this paper, although they are, generally, different functions.) has the properties given in "Finite strain" section and $\phi_{(I)}$ depends on the spectral invariants $\lambda_{i}$ and $a_{i}$.

The weighted Cauchy stress takes the form

$$
J \boldsymbol{T}=\sum_{i=1}^{3}\left(\lambda_{i}^{*} \frac{\partial W_{(T)}}{\partial \lambda_{i}^{*}}-p^{*}\right) \boldsymbol{v}_{i} \otimes \boldsymbol{v}_{i}+\sum_{i \neq j}^{3} \frac{2 a_{i} a_{j} \lambda_{i}^{*} \lambda_{j}^{*}}{\lambda_{i}^{* 2}-\lambda_{j}^{* 2}}\left(\frac{\partial W_{(T)}}{\partial \zeta_{i}}-\frac{\partial W_{(T)}}{\partial \zeta_{j}}\right) \boldsymbol{v}_{i} \otimes \boldsymbol{v}_{j}
$$

\section{Two preferred direction elastic solid}

Consider an elastic material with preferred unit directions $\boldsymbol{a}$ and $\boldsymbol{b}$, where the unit vectors $\boldsymbol{a}$ and $\boldsymbol{b}$ are independent. The strain energy

$$
W_{(P)}=\bar{W}_{P}(\boldsymbol{U}, \boldsymbol{a} \otimes \boldsymbol{a}, \boldsymbol{b} \otimes \boldsymbol{b})=W_{P}(\boldsymbol{U}, \boldsymbol{a}, \boldsymbol{b})=W_{P}\left(\boldsymbol{Q U} \boldsymbol{U} \boldsymbol{Q}^{T}, \boldsymbol{Q} \boldsymbol{a}, \boldsymbol{Q b}\right) .
$$

Hence, we can express $W_{(P)}$ in terms of the spectral invariants ${ }^{17,30}$

$$
\lambda_{i}, \quad a_{i}, \quad \iota_{i}=\boldsymbol{u}_{i} \cdot \boldsymbol{b}=\boldsymbol{Q} \boldsymbol{u}_{i} \cdot \boldsymbol{Q} \boldsymbol{b} .
$$

We note that in view of (57) and the relations

$$
\sum_{i=1} \gamma_{i}=1, \quad \gamma_{i}=\iota_{i}^{2}
$$

only 7 of the 9 invariants in (74) are independent and they formed the minimal/irreducible integrity basis ${ }^{30,32}$.

Infinitesimal strain. Modifying the work of Shariff and Bustamante ${ }^{30}$, we have the strain energy

$$
\begin{aligned}
W_{(P)}= & W_{p}\left(\boldsymbol{E}^{*}, h\right)=b_{1} \operatorname{tr} \boldsymbol{E}^{* 2}+b_{2} \boldsymbol{a} \cdot \boldsymbol{E}^{* 2} \boldsymbol{a}+b_{3}\left(\boldsymbol{a} \cdot \boldsymbol{E}^{*} \boldsymbol{a}\right)^{2}+b_{4} \boldsymbol{b} \cdot \boldsymbol{E}^{* 2} \boldsymbol{b}+b_{5}\left(\boldsymbol{b} \cdot \boldsymbol{E}^{*} \boldsymbol{b}\right)^{2} \\
& +b_{6}(\boldsymbol{a} \cdot \boldsymbol{b}) \boldsymbol{a} \cdot \boldsymbol{E}^{* 2} \boldsymbol{b}+b_{7}\left[(\boldsymbol{a} \cdot \boldsymbol{b}) \boldsymbol{a} \cdot \boldsymbol{E}^{*} \boldsymbol{b}\right]^{2}+b_{8}\left(\boldsymbol{a} \cdot \boldsymbol{E}^{*} \boldsymbol{a}\right)\left(\boldsymbol{b} \cdot \boldsymbol{E}^{*} \boldsymbol{b}\right)+b_{9}(\boldsymbol{a} \cdot \boldsymbol{b})\left(\boldsymbol{a} \cdot \boldsymbol{E}^{*} \boldsymbol{b}\right)\left(\boldsymbol{a} \cdot \boldsymbol{E}^{*} \boldsymbol{a}\right) \\
& +b_{10}(\boldsymbol{a} \cdot \boldsymbol{b})\left(\boldsymbol{a} \cdot \boldsymbol{E}^{*} \boldsymbol{b}\right)\left(\boldsymbol{b} \cdot \boldsymbol{E}^{*} \boldsymbol{b}\right)+b_{11} h\left(\boldsymbol{a} \cdot \boldsymbol{E}^{*} \boldsymbol{a}\right)+b_{12} h\left(\boldsymbol{b} \cdot \boldsymbol{E}^{*} \boldsymbol{b}\right)+b_{13} h(\boldsymbol{a} \cdot \boldsymbol{b})\left(\boldsymbol{a} \cdot \boldsymbol{E}^{*} \boldsymbol{b}\right)+\frac{\chi}{2} h^{2} .
\end{aligned}
$$

The mean hydrostatic stress is

$$
\frac{\operatorname{tr} \boldsymbol{T}}{3}=\frac{\partial W_{p}}{\partial h}\left(\boldsymbol{E}^{*}, h\right)=b_{11}\left(\boldsymbol{a} \cdot \boldsymbol{E}^{*} \boldsymbol{a}\right)+b_{12}\left(\boldsymbol{b} \cdot \boldsymbol{E}^{*} \boldsymbol{b}\right)+b_{13}(\boldsymbol{a} \cdot \boldsymbol{b})\left(\boldsymbol{a} \cdot \boldsymbol{E}^{*} \boldsymbol{b}\right)+h \chi .
$$

The bulk modulus

$$
\chi=\frac{\partial^{2} W_{p}}{\partial h^{2}}\left(\boldsymbol{E}^{*}, h\right)
$$


Mechanically equivalent material. For a mechanically equivalent material we simply let

$$
b_{2}=b_{4}, \quad b_{3}=b_{5}, \quad b_{9}=b_{10}, \quad b_{11}=b_{12},
$$

and we have only 10 material constants [In Shariff and Bustamante ${ }^{30}$, they mistakenly evaluate 11 material constants.] to characterize its mechanical behaviour. Hence, we have, the strain energy

$$
\begin{aligned}
W_{(M)}=W_{M}\left(\boldsymbol{E}^{*}, h\right)= & c_{1} \operatorname{tr} \boldsymbol{E}^{* 2}+c_{2}\left[\boldsymbol{a} \cdot \boldsymbol{E}^{* 2} \boldsymbol{a}+\boldsymbol{b} \cdot \boldsymbol{E}^{* 2} \boldsymbol{b}\right]+c_{3}\left[\left(\boldsymbol{a} \cdot \boldsymbol{E}^{*} \boldsymbol{a}\right)^{2}+\left(\boldsymbol{b} \cdot \boldsymbol{E}^{*} \boldsymbol{b}\right)^{2}\right] \\
& +c_{4}(\boldsymbol{a} \cdot \boldsymbol{b}) \boldsymbol{a} \cdot \boldsymbol{E}^{* 2} \boldsymbol{b}+c_{5}\left[(\boldsymbol{a} \cdot \boldsymbol{b}) \boldsymbol{a} \cdot \boldsymbol{E}^{*} \boldsymbol{b}\right]^{2}+c_{6}\left(\boldsymbol{a} \cdot \boldsymbol{E}^{*} \boldsymbol{a}\right)\left(\boldsymbol{b} \cdot \boldsymbol{E}^{*} \boldsymbol{b}\right) \\
& +c_{7}\left[(\boldsymbol{a} \cdot \boldsymbol{b})\left(\boldsymbol{a} \cdot \boldsymbol{E}^{*} \boldsymbol{b}\right)\left(\boldsymbol{a} \cdot \boldsymbol{E}^{*} \boldsymbol{a}\right)+(\boldsymbol{a} \cdot \boldsymbol{b})\left(\boldsymbol{a} \cdot \boldsymbol{E}^{*} \boldsymbol{b}\right)\left(\boldsymbol{b} \cdot \boldsymbol{E}^{*} \boldsymbol{b}\right)\right] \\
& \left.+c_{8}\left[h\left(\boldsymbol{a} \cdot \boldsymbol{E}^{*} \boldsymbol{a}\right)+h\left(\boldsymbol{b} \cdot \boldsymbol{E}^{*} \boldsymbol{b}\right)\right]+c_{9} h(\boldsymbol{a} \cdot \boldsymbol{b})\left(\boldsymbol{a} \cdot \boldsymbol{E}^{*} \boldsymbol{b}\right)\right]+\frac{\chi}{2} h^{2},
\end{aligned}
$$

where $c_{\alpha}(\alpha=1,2, \ldots 9)$ are ground state material constants.

Orthotropic elastic solid. In the case of an orthotropic material, where the preferred directions $\boldsymbol{a}$ and $\boldsymbol{b}$ are orthogonal, we have

$$
\begin{aligned}
W_{(O)}= & W_{O}\left(\boldsymbol{E}^{*}, h\right)=d_{1} \operatorname{tr} \boldsymbol{E}^{* 2}+d_{2} \boldsymbol{a} \cdot \boldsymbol{E}^{* 2} \boldsymbol{a}+d_{3}\left(\boldsymbol{a} \cdot \boldsymbol{E}^{*} \boldsymbol{a}\right)^{2}+d_{4} \boldsymbol{b} \cdot \boldsymbol{E}^{* 2} \boldsymbol{b}+d_{5}\left(\boldsymbol{b} \cdot \boldsymbol{E}^{*} \boldsymbol{b}\right)^{2} \\
& +d_{6}\left(\boldsymbol{a} \cdot \boldsymbol{E}^{*} \boldsymbol{a}\right)\left(\boldsymbol{b} \cdot \boldsymbol{E}^{*} \boldsymbol{b}\right)++d_{7} h\left(\boldsymbol{a} \cdot \boldsymbol{E}^{*} \boldsymbol{a}\right)+d_{8} h\left(\boldsymbol{b} \cdot \boldsymbol{E}^{*} \boldsymbol{b}\right)+\frac{\chi}{2} h^{2},
\end{aligned}
$$

where $d_{1}, d_{2}, \ldots d_{8}$ are ground state constants.

Finite strain. Following the work of sections "Isotropic" and "Transversely Isotropic with a unit preferred direction $\boldsymbol{a}$ " sections, we propose the strain energy function

$$
\begin{aligned}
W_{(P)}= & b_{1} \sum_{i=1}^{3} f_{1}\left(\lambda_{i}^{*}\right) f_{2}\left(\lambda_{i}^{*}\right)+b_{2} \sum_{i=1}^{3} \zeta_{i} f_{3}\left(\lambda_{i}^{*}\right) f_{4}\left(\lambda_{i}^{*}\right)+b_{3} \sum_{i=1}^{3} \zeta_{i} f_{5}\left(\lambda_{i}^{*}\right) \sum_{i=1}^{3} \zeta_{i} f_{6}\left(\lambda_{i}^{*}\right) \\
& +b_{4} \sum_{i=1}^{3} \gamma_{i} f_{7}\left(\lambda_{i}^{*}\right) f_{8}\left(\lambda_{i}^{*}\right)+b_{5} \sum_{i=1}^{3} \gamma_{i} f_{9}\left(\lambda_{i}^{*}\right) \sum_{i=1}^{3} \gamma_{i} f_{10}\left(\lambda_{i}^{*}\right)+b_{6} \sum_{i=1}^{3} \eta_{i} f_{11}\left(\lambda_{i}^{*}\right) f_{12}\left(\lambda_{i}^{*}\right) \\
& +b_{7} \sum_{i=1}^{3} \eta_{i} f_{13}\left(\lambda_{i}^{*}\right) \sum_{i=1}^{3} \eta_{i} f_{14}\left(\lambda_{i}^{*}\right)+b_{8} \sum_{i=1}^{3} \zeta_{i} f_{15}\left(\lambda_{i}^{*}\right) \sum_{i=1}^{3} \gamma_{i} f_{16}\left(\lambda_{i}^{*}\right) \\
& +b_{9} \sum_{i=1}^{3} \eta_{i} f_{17}\left(\lambda_{i}^{*}\right) \sum_{i=1}^{3} \zeta_{i} f_{18}\left(\lambda_{i}^{*}\right)+b_{10} \sum_{i=1}^{3} \eta_{i} f_{19}\left(\lambda_{i}^{*}\right) \sum_{i=1}^{3} \gamma_{i} f_{20}\left(\lambda_{i}^{*}\right) \\
& +b_{11} g_{1}(J) \sum_{i=1}^{3} \zeta_{i} f_{21}\left(\lambda_{i}^{*}\right)+b_{12} g_{2}(J) \sum_{i=1}^{3} \gamma_{i} f_{22}\left(\lambda_{i}^{*}\right) \\
& +b_{13} g_{3}(J) \sum_{i=1}^{3} \eta_{i} f_{23}\left(\lambda_{i}^{*}\right)+\frac{\chi}{2} g_{4}(J) g_{5}(J)+\phi_{(I)},
\end{aligned}
$$

where $\eta_{i}=(\boldsymbol{a} \cdot \boldsymbol{b}) a_{i} \iota_{i}, \phi_{(I)}$ has the properties given in "Finite strain" section and is a function of $\lambda_{i}, a_{i}$ and $\iota_{i}$.

The weighted Cauchy stress takes the form

$$
\begin{aligned}
J \boldsymbol{T}= & \sum_{i=1}^{3}\left(\lambda_{i}^{*} \frac{\partial W_{(T)}}{\partial \lambda_{i}^{*}}-p^{*}\right) \boldsymbol{v}_{i} \otimes \boldsymbol{v}_{i}+\sum_{i \neq j}^{3} \frac{2 \lambda_{i}^{*} \lambda_{j}^{*}}{\lambda_{i}^{* 2}-\lambda_{j}^{* 2}}\left\{\left(\frac{\partial W_{(T)}}{\partial \zeta_{i}}-\frac{\partial W_{(T)}}{\partial \zeta_{j}}\right) a_{i} a_{j}\right. \\
& \left.+\left(\frac{\partial W_{(T)}}{\partial \gamma_{i}}-\frac{\partial W_{(T)}}{\partial \gamma_{j}}\right) \iota_{i} \iota_{j}+\frac{\boldsymbol{a} \cdot \boldsymbol{b}}{2}\left(\frac{\partial W_{(T)}}{\partial \eta_{i}}-\frac{\partial W_{(T)}}{\partial \eta_{j}}\right)\left(a_{i} \iota_{j}+a_{j} \iota_{i}\right)\right\} \boldsymbol{v}_{i} \otimes \boldsymbol{v}_{j} .
\end{aligned}
$$


Mechanical equivalent elastic solid.

$$
\begin{aligned}
W_{(M)}= & c_{1} \sum_{i=1}^{3} f_{1}\left(\lambda_{i}^{*}\right) f_{2}\left(\lambda_{i}^{*}\right)+c_{2} \sum_{i=1}^{3}\left(\zeta_{i}+\gamma_{i}\right) f_{3}\left(\lambda_{i}^{*}\right) f_{4}\left(\lambda_{i}^{*}\right) \\
& +c_{3}\left\{\sum_{i=1}^{3} \zeta_{i} f_{5}\left(\lambda_{i}^{*}\right) \sum_{i=1}^{3} \zeta_{i} f_{6}\left(\lambda_{i}^{*}\right)+\sum_{i=1}^{3} \gamma_{i} f_{5}\left(\lambda_{i}^{*}\right) \sum_{i=1}^{3} \gamma_{i} f_{6}\left(\lambda_{i}^{*}\right)\right\}+c_{4} \sum_{i=1}^{3} \eta_{i} f_{7}\left(\lambda_{i}^{*}\right) f_{8}\left(\lambda_{i}^{*}\right) \\
& +c_{5} \sum_{i=1}^{3} \eta_{i} f_{9}\left(\lambda_{i}^{*}\right) \sum_{i=1}^{3} \eta_{i} f_{10}\left(\lambda_{i}^{*}\right)+c_{6} \sum_{i=1}^{3} \zeta_{i} f_{11}\left(\lambda_{i}^{*}\right) \sum_{i=1}^{3} \gamma_{i} f_{11}\left(\lambda_{i}^{*}\right) \\
& +c_{7} \sum_{i=1}^{3} \eta_{i} f_{12}\left(\lambda_{i}^{*}\right) \sum_{i=1}^{3}\left(\zeta_{i}+\gamma_{i}\right) f_{13}\left(\lambda_{i}^{*}\right)+c_{8} g_{1}(J) \sum_{i=1}^{3}\left(\zeta_{i}+\gamma_{i}\right) f_{14}\left(\lambda_{i}^{*}\right) \\
& +c_{9} g_{2}(J) \sum_{i=1}^{3} \eta_{i} f_{15}\left(\lambda_{i}^{*}\right)+\frac{\chi}{2} g_{4}(J) g_{5}(J)+\phi_{(I)} .
\end{aligned}
$$

An orthotropic strain energy function can be easily obtained from (82) by letting $\eta_{i}=0$.

\section{General anisotropy}

Consider the strain energy $W_{(G)}$ that depends of the 4 th order classical stiffness tensor $\mathbb{C}$, i.e.,

$$
W_{(G)}=W_{g}(\mathbb{C}, \boldsymbol{U}) .
$$

Note that $W_{g}$ must be invariant with respect to the rotation $\boldsymbol{Q}$, i.e.

$$
W_{g}(\mathbb{C}, \boldsymbol{U})=W_{g}\left(\overline{\mathbb{C}}, \boldsymbol{Q U} \boldsymbol{Q}^{T}\right),
$$

where $^{43}$

$$
\overline{\mathbb{C}}=\left[\left(\left(\mathbb{C}: \boldsymbol{Q}^{T}\right): \boldsymbol{Q}^{T}\right): \boldsymbol{Q}^{T}\right] \mid \boldsymbol{Q}^{T},
$$

and, the operations : and are defined as follows

$$
\begin{aligned}
& a_{1} \otimes a_{2} \otimes a_{3} \otimes a_{4}: b_{1} \otimes b_{2}=\left(b_{1} \cdot a_{2}\right) a_{1} \otimes a_{3} \otimes a_{4} \otimes b_{2}, \\
& a_{1} \otimes a_{2} \otimes a_{3} \otimes a_{4} \mid b_{1} \otimes b_{2}=\left(b_{1} \cdot a_{1}\right) b_{2} \otimes a_{2} \otimes a_{3} \otimes a_{4} .
\end{aligned}
$$

We also have the definition

$$
\left(a_{1} \otimes a_{2} \otimes a_{3} \otimes a_{4}\right)\left(b_{1} \otimes b_{2}\right)=\left(a_{1} \otimes a_{2}\right)\left(a_{3} \cdot b_{1}\right)\left(a_{4} \cdot b_{2}\right) .
$$

In view of (87),(88) and (89), we obtain

$$
\overline{\mathbb{C}}=\sum_{i, j, k, l} c_{i j k l} \boldsymbol{Q} \boldsymbol{u}_{i} \otimes \boldsymbol{Q} \boldsymbol{u}_{j} \otimes \boldsymbol{Q} \boldsymbol{u}_{k} \otimes \boldsymbol{Q} \boldsymbol{u}_{l},
$$

where

$$
c_{i j k l}=\boldsymbol{u}_{i} \cdot\left[\mathbb{C}\left(\boldsymbol{u}_{k} \otimes \boldsymbol{u}_{l}\right)\right] \boldsymbol{u}_{j}
$$

From (87) and (91), we have

$$
c_{i j k l}=\boldsymbol{u}_{i} \cdot\left[\mathbb{C}\left(\boldsymbol{u}_{k} \otimes \boldsymbol{u}_{l}\right)\right] \boldsymbol{u}_{j}=\boldsymbol{Q} \boldsymbol{u}_{i} \cdot\left[\overline{\mathbb{C}}\left(\boldsymbol{Q} \boldsymbol{u}_{k} \otimes \boldsymbol{Q} \boldsymbol{u}_{l}\right)\right] \boldsymbol{Q} \boldsymbol{u}_{j},
$$

for all rotation $Q$ and this implies that $c_{i j k l}$ are invariants. Hence, the strain energy $W_{(G)}$ can be expressed in terms of the invariants

$$
c_{i j k l}, \quad \lambda_{i}
$$

with the symmetrical properties

$$
c_{i j k l}=c_{j i k l}=c_{k l i j}=c_{i j l k} .
$$

Due to the symmetrical property (95), only 24 of the invariants in (94) are independent. These 24 invariants are elements of the irreducible/minimal integrity basis. Hence, all other invariants (see, for example, reference ${ }^{44}$ ) can be expressed in terms of the 24 independent spectral invariants.

Infinitesimal strain. The strain energy for a general anisotropic elastic solid is 


$$
\begin{aligned}
W_{(G)} & =\frac{1}{2}(\operatorname{tr}[\mathbb{C} \boldsymbol{E}] \boldsymbol{E})=\frac{1}{2} \operatorname{tr}\left(\left[\mathbb{C}\left(\boldsymbol{E}^{*}+\frac{h}{3} \boldsymbol{I}\right]\left(\boldsymbol{E}^{*}+\frac{h}{3} \boldsymbol{I}\right)\right)\right. \\
& =\frac{1}{2}\left(\operatorname{tr}\left[\mathbb{C} \boldsymbol{E}^{*}\right] \boldsymbol{E}^{*}+\frac{2 h}{3} \operatorname{tr}\left[\mathbb{C} \boldsymbol{E}^{*}\right] \boldsymbol{I}+\frac{h^{2}}{9} \operatorname{tr}[\mathbb{C} \boldsymbol{I}] \boldsymbol{I}\right) .
\end{aligned}
$$

The infinitesimal stress takes the form

$$
\boldsymbol{T}=\mathbb{C} \boldsymbol{E}^{*}+\frac{h}{3} \mathbb{C} \boldsymbol{I}
$$

The hydrostatic stress is

$$
\frac{\operatorname{tr} T}{3}=\frac{\operatorname{tr} \mathbb{C} E^{*}}{3}+h \frac{\operatorname{tr} \mathbb{C I}}{9} .
$$

From (98), we have the ground-state bulk modulus

$$
\chi=\frac{\operatorname{tr} \mathbb{C} I}{9} .
$$

In terms of spectral expressions, we have,

$$
\begin{gathered}
W_{(G)}=\sum_{i, r=1}^{3}\left\{c_{i i r r}\left[\frac{e_{i}^{*} e_{r}^{*}}{2}+\frac{h e_{r}^{*}}{3}+\frac{h^{2}}{18}\right]\right\}, \\
\mathbb{C}=\sum_{i, j, k, l} c_{i j k l} \boldsymbol{u}_{i} \otimes \boldsymbol{u}_{j} \otimes \boldsymbol{u}_{k} \otimes \boldsymbol{u}_{l}
\end{gathered}
$$

and the ground-state bulk modulus

$$
\chi=\frac{1}{3} \sum_{i, r=1}^{3} c_{i i r r}
$$

Finite strain. In view of (100), we propose a (not the) general finite strain energy for a general anisotropic material, i.e.

$$
W_{(G)}=\sum_{i, r=1}^{3} c_{i i r r}\left(\frac{f_{1}\left(\lambda_{i}^{*}\right) f_{2}\left(\lambda_{r}^{*}\right)}{2}+\frac{g_{1}(J) f_{3}\left(\lambda_{r}^{*}\right)}{3}+\frac{g_{2}(J) g_{3}(J)}{18}\right)+\phi_{(I)},
$$

where $\phi_{(I)}$ depends on the spectral invariants $\lambda_{i}$ and $c_{i j k l}$. For example, when specialized to a transversely isotropic material, we have from (71)

$$
\begin{aligned}
W_{(G)}= & W_{(T)}=\bar{a}_{1} \sum_{i=1}^{3} f_{1}\left(\lambda_{i}^{*}\right) f_{2}\left(\lambda_{i}^{*}\right)+a_{(2)} g_{2}(J) g_{3}(J)+\bar{a}_{3} \sum_{i=1}^{3} \zeta_{i} f_{1}\left(\lambda_{i}^{*}\right) f_{2}\left(\lambda_{i}^{*}\right) \\
& +\bar{a}_{4}\left(\sum_{i=1}^{3} \zeta_{i} f_{1}\left(\lambda_{i}^{*}\right)\right)\left(\sum_{i=1}^{3} \zeta_{i} f_{2}\left(\lambda_{i}^{*}\right)\right)+a_{(5)} g_{1}(J) \sum_{i=1}^{3} \zeta_{i} f_{3}\left(\lambda_{i}^{*}\right)+\phi_{(I)} .
\end{aligned}
$$

In this case the spectral components take the form

$$
\begin{aligned}
c_{i j k l}= & \bar{a}_{1}\left(\delta_{i k} \delta_{j l}+\delta_{j k} \delta_{i l}\right)+2 \bar{a}_{2} \delta_{i j} \delta_{k l}+\frac{\bar{a}_{3}}{2}\left(a_{i} a_{k} \delta_{j l}+a_{i} a_{l} \delta_{j k}+a_{j} a_{k} \delta_{i l}+a_{j} a_{l} \delta_{i k}\right) \\
& +2 \bar{a}_{4} a_{i} a_{j} a_{k} a_{l}+\bar{a}_{5}\left(a_{k} a_{l} \delta_{i j}+a_{i} a_{j} \delta_{k l}\right) .
\end{aligned}
$$

As mentioned earlier, it is important that $W_{(G)}$ satisfies the $P$-property. It is clear that, in view of (92), that the symmetrical part of the $P$-property is satisfied. We now show that $W_{(G)}$ has a unique value when two or more of the principal stretches have a same value. Consider the case when $\lambda_{1}^{*}=\lambda_{2}^{*}=\lambda$. In this case the principal directions $\boldsymbol{u}_{1}$ and $\boldsymbol{u}_{2}$ are not unique but $\boldsymbol{u}_{3}$ has a unique direction. We then have

$$
W_{(G)}=W_{1}+W_{2}+W_{3},
$$

where

$$
\begin{aligned}
W_{1}= & \frac{1}{2}\left\{\left(c_{1111}+c_{2222}+c_{1122}+c_{2211}\right) f_{1}(\lambda) f_{2}(\lambda)\right. \\
& \left.+\left(c_{1133}+c_{2233}\right) f_{1}(\lambda) f_{2}\left(\lambda_{3}\right)+\left(c_{3311}+c_{3322}\right) f_{1}\left(\lambda_{3}\right) f_{2}(\lambda)\right\}
\end{aligned}
$$




$$
\begin{gathered}
W_{2}=\frac{g_{1}(J)}{3}\left\{\sum_{i=1}^{3}\left(c_{i i 11}+c_{i i 22}\right) f_{3}(\lambda)+c_{i i 33} f_{3}\left(\lambda_{3}\right)\right\}, \\
W_{3}=\frac{1}{18} \operatorname{tr}(\mathbb{C} \boldsymbol{I}) g_{2}(J) g_{3}(J) .
\end{gathered}
$$

Making use of the relations

$$
\begin{aligned}
& \operatorname{tr}\left[\mathbb{C}\left(\boldsymbol{u}_{3} \otimes \boldsymbol{u}_{3}\right)\right]=c_{1133}+c_{2233}+c_{3333}=\operatorname{tr}\left[(\mathbb{C} \boldsymbol{I})\left(\boldsymbol{u}_{3} \otimes \boldsymbol{u}_{3}\right)\right]=c_{3311}+c_{3322}+c_{3333}, \\
& \operatorname{tr}[\mathbb{C} \boldsymbol{I}]=\sum_{i, r=1}^{3} c_{i i r r},
\end{aligned}
$$

we have

$$
\begin{gathered}
W_{1}=\frac{1}{2}\left(A f_{1}(\lambda) f_{2}(\lambda)+B\left[f_{1}\left(\lambda_{3}\right) f_{2}(\lambda)+f_{1}(\lambda) f_{2}\left(\lambda_{3}\right)\right]\right), \\
W_{2}=\frac{g_{1}(J)}{3}\left((A+B) f_{3}(\lambda)+\left(B+c_{3333}\right) f_{3}\left(\lambda_{3}\right)\right),
\end{gathered}
$$

where

$$
A=\operatorname{tr}\left[\mathbb{C} \boldsymbol{I}-\mathbb{C}\left(\boldsymbol{u}_{3} \otimes \boldsymbol{u}_{3}\right)-\mathbb{C} \boldsymbol{I}\left(\boldsymbol{u}_{3} \otimes \boldsymbol{u}_{3}\right)\right]+2 c_{3333}, \quad B=\operatorname{tr}\left[\mathbb{C} \boldsymbol{I}\left(\boldsymbol{u}_{3} \otimes \boldsymbol{u}_{3}\right)\right]-c_{3333} .
$$

It is clear from (109),(111), (112) and (113) that $W_{(G)}$ has a unique value when $\lambda_{1}^{*}=\lambda_{2}^{*}=\lambda$, since it is independent of the eigenvectors $\boldsymbol{u}_{1}$ and $\boldsymbol{u}_{2}$. Following the above method, it is straightfoward to show that $W_{(G)}$ has a unique value when any two of the principal stretches have a same value. In the case when $\lambda_{1}^{*}=\lambda_{2}^{*}=\lambda_{3}^{*}=\lambda$, the value of $W_{(G)}$ is unique since, in this case,

$$
W_{(G)}=\operatorname{tr}[\mathbb{C I}]\left\{\frac{1}{2} f_{1}(\lambda) f_{2}(\lambda)+\frac{1}{3} g_{1}(J) f_{3}(\lambda)+\frac{1}{18} g_{2}(J) g_{3}(J)\right\} .
$$

The weighted Cauchy stress is

$$
J \boldsymbol{T}=\sum_{i=1}^{3}\left(\lambda_{i}^{*} \frac{\partial W_{(G)}}{\partial \lambda_{i}^{*}}-p^{*}\right) \boldsymbol{v}_{i} \otimes \boldsymbol{v}_{i}+\sum_{i \neq j}^{3} \frac{4 \lambda_{i}^{*} \lambda_{j}^{*}}{\lambda_{i}^{* 2}-\lambda_{j}^{* 2}} \kappa_{i j} \boldsymbol{v}_{i} \otimes \boldsymbol{v}_{j},
$$

where

$$
\kappa_{i j}=\frac{\partial W_{(G)}}{\partial c_{i i i i}} c_{i j i i}-\frac{\partial W_{(G)}}{\partial c_{j j j j}} c_{i j j j}+\sum_{r \neq i} \frac{\partial W_{(G)}}{\partial c_{i i r r}} c_{i j r r}-\sum_{r \neq j}^{3} \frac{\partial W_{(G)}}{\partial c_{j j r r}} c_{i j r r} .
$$

\section{Example of specific forms of $f_{\alpha}$ and $g_{\alpha}$ used in experimental fitting}

In this section, we suggest specific forms of the strain functions $f_{\alpha}$ and $g_{\alpha}$ to fit experiment data. We strongly emphasize that we are not interested in constructing the (or an) optimal form of $f_{\alpha}$ and $g_{\alpha}$ for a particular material; we are only interested in giving examples of specific forms of the proposed strain functions that can be used to fit experimental data. Constructing an optimal form of the strain functions for a particular material similar to the previous work of Shariff ${ }^{12,14,37}$ will be done in the near future. We also emphasize that the specific forms are mainly constructed via visual curve fitting. Since, we are dealing with many types of isotropic and anisotropic materials, curve fitting exercises (such as those found in references ${ }^{45-47}$ for isotropic solids only), for all the isotropic/anisotropic solids mentioned below require a considerable amount of work and analysis, and it is outside the scope of this paper.

Only strain energy functions with $\phi_{(I)}=0$ are discussed in this section.

Isotropic. In the case of a compressible material, we use the simple strain functions based on the Hencky strain energy function ${ }^{40}$

$$
f_{1}(x)=f_{2}(x)=g_{1}(x)=g_{2}(x)=\ln x,
$$

to fit the simple tension data of an isotropic polyurethane foam material used in Blatz and $\mathrm{Ko}^{48}$ experiment, where four sets of data are used. The nominal axial stress in the 3 -direction is

$$
t_{3}=\lambda_{3}^{*} \frac{\partial W_{(I)}}{\partial \lambda_{3}^{*}}-\lambda_{1}^{*} \frac{\partial W_{(I)}}{\partial \lambda_{1}^{*}}
$$

The values of the lateral stretch $\lambda_{1}=\lambda_{2}$ is obtained in terms of $\lambda_{3}$ via the zero lateral stress condition, i.e., 


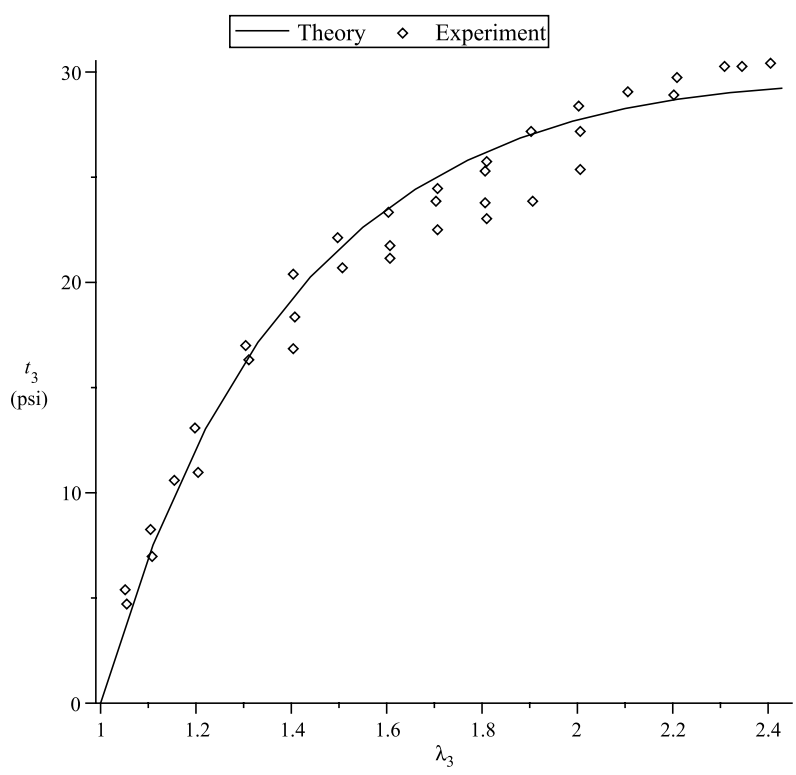

Figure 1. Axial nominal stress $t_{3}$ versus axial stretch $\lambda_{3} . \mu=32$ and $v=0.25$.

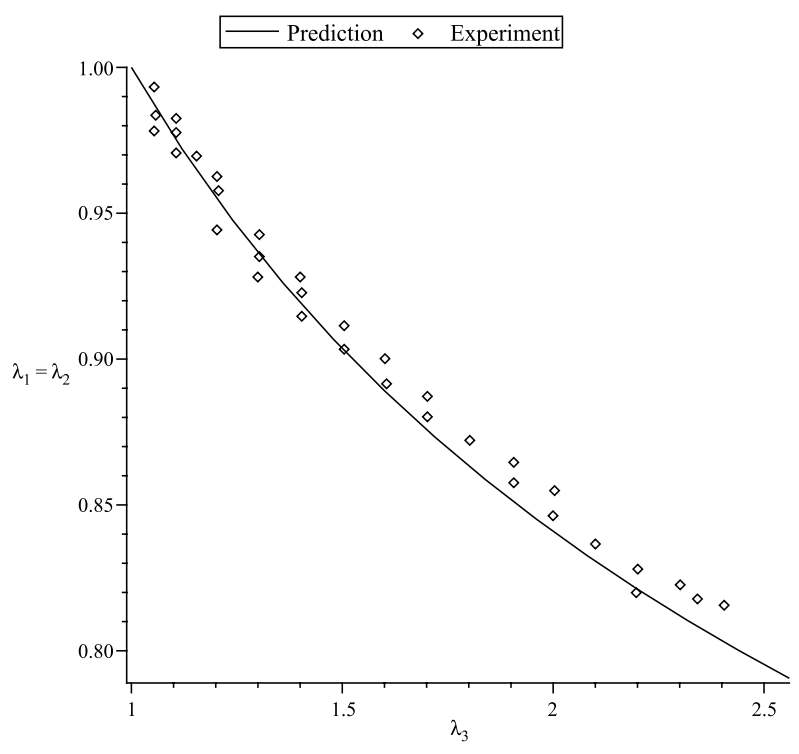

Figure 2. Prediction of the Lateral stretch $\lambda_{1}=\lambda_{2}$ versus axial stretch $\lambda_{3}$.

$$
\lambda_{1}^{*} \frac{\partial W_{(I)}}{\partial \lambda_{1}^{*}}=\lambda_{2}^{*} \frac{\partial W_{(I)}}{\partial \lambda_{2}^{*}} \quad \text { or } \quad \lambda_{1}^{*} \frac{\partial W_{(I)}}{\partial \lambda_{1}^{*}}=p^{*}
$$

We use the ground-state values

$$
\mu=32 \mathrm{psi}, \quad v=0.25
$$

to fit the simple tension data in Fig. 1; these are the same values that are obtained in Blatz and $\mathrm{Ko}^{48}$ experiment. Fig. 1 shows that our theory reasonably fit the nominal stress vs. axial stretch curve and the behaviour of the lateral stretch $\lambda_{1}=\lambda_{2}$ in terms of the axial stretch $\lambda_{3}$ is predicted quite well in Fig. 2.

For incompressible materials $(J=1)$, we give, below, specific forms for the strain functions to fit the experimental data of Treloar ${ }^{49}$ and Jones and Treloar ${ }^{50}$. In the case of Treloar's ${ }^{49}$ data, we use the strain functions ${ }^{37}$ 

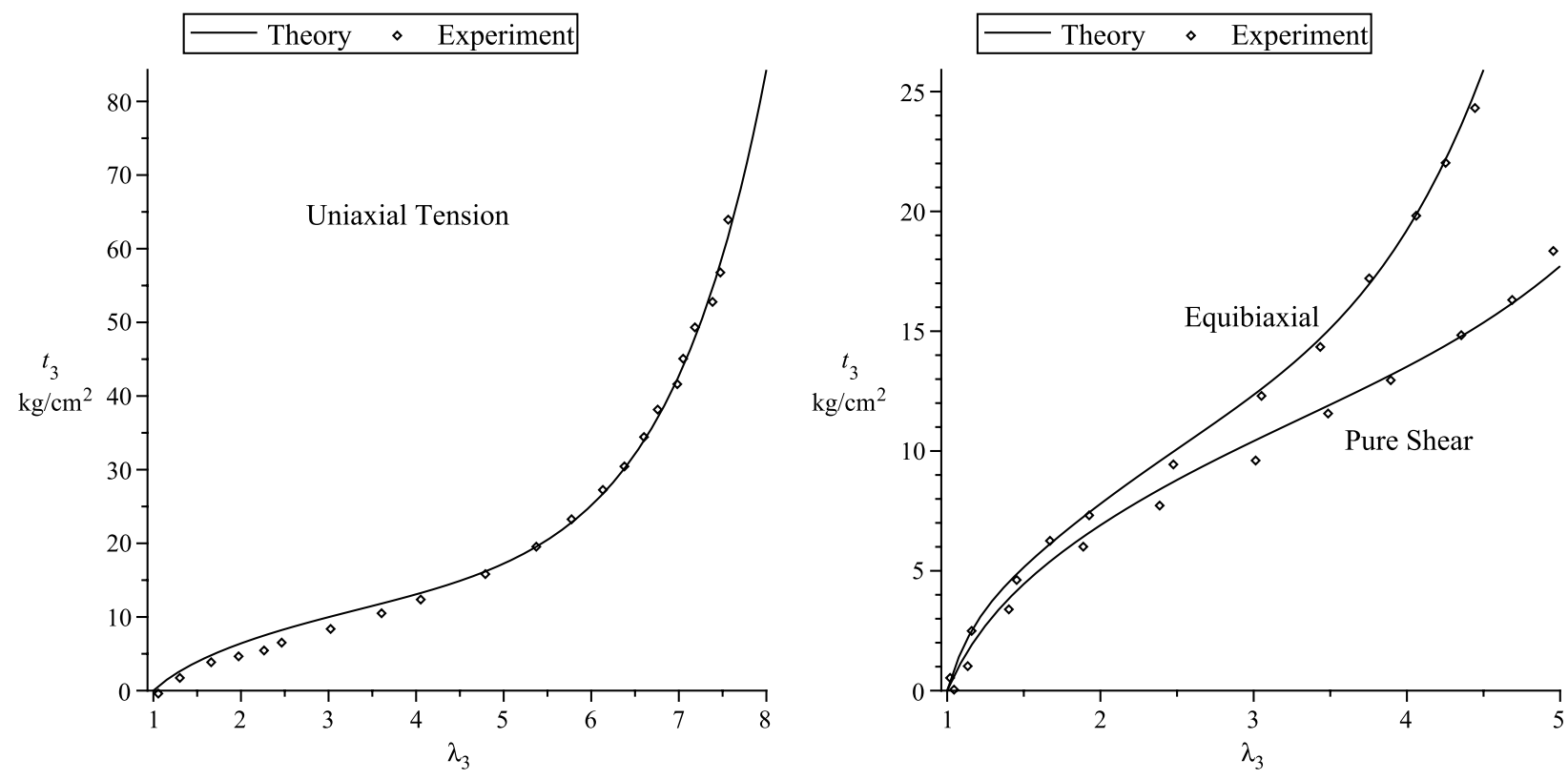

Figure 3. Comparison of theoretical curves with the Treloar's ${ }^{49}$ experimental data.

$$
\begin{aligned}
f_{1}= & f_{2}, \quad f_{1}^{2}(x)=r(x)=\ln (x)^{2}+3\left(1.37334 \psi_{1}(x)+0.471163 \times 10^{-1} \psi_{2}\right. \\
& \left.+0.841383 \times 10^{-4} \psi_{3}\right) \geq 0
\end{aligned}
$$

to fit Treloar $^{49}$ experimental data, where

$$
\psi_{1}=\int_{1}^{x} \frac{e^{1-s}}{s} d s+x-2 \ln x-1, \quad \psi_{2}=\int_{1}^{x} \frac{e^{s-1}}{s}-x+1, \quad \psi_{3}=\int_{1}^{x} \frac{(s-1)^{3}}{s^{4.6}} d s .
$$

In uniaxial, pure shear and equibiaxial extensions, the principal nominal stress $t_{3}$ can be simply expressed as

$$
t_{3}=\frac{\lambda_{3} r^{\prime}\left(\lambda_{3}\right)-\frac{1}{\lambda_{3}^{m}} r^{\prime}\left(\frac{1}{\lambda_{3}^{m}}\right)}{\lambda_{3}}
$$

where $m=0.5,1,2$ corresponds, respectively, to uniaxial, pure shear and equibiaxial deformatons. It is clear in Fig. 3, that the theoretical curves for the three different deformations fit the experiment data very well when the shear modulus has the value $\mu=\frac{11.009}{3} \mathrm{~kg} / \mathrm{cm}^{2}$.

To fit Jones and $\operatorname{Treloar}^{50}$ biaxial experimental data, we use $\mu=0.4(\mathrm{MPa})$ and the strain functions

$$
f_{1}=f_{2}, \quad f_{1}^{2}(x)=r(x)=\ln (x)^{2}+2.4669 \psi_{1}(x)+0.3771 \psi_{2} \geq 0 .
$$

The biaxial deformation experiment require the stress difference

$$
\sigma_{1}-\sigma_{2}=\mu\left(\lambda_{1} r^{\prime}\left(\lambda_{1}\right)-\lambda_{2} r^{\prime}\left(\lambda_{2}\right)\right)
$$

where $\sigma_{1}$ and $\sigma_{2}$ are principal Cauchy stresses. Fig. 4 indicates that the strain functions (124) fits the experiment extremely well.

Transversely isotropic. We compare our theory with the axial compression experiment of Jin et al. ${ }^{51}$ on compressible rectangular slabs of transversely isotropic Marcellus shale. Although the measured experimental strains are infinitesimal, the stress-strain behaviour is very mildly nonlinear ${ }^{42}$. Since, the strains are infinitesimal, the Cauchy and nominal stress are indistinguishable. The nominal stress-strain relation

$$
t_{3}=\frac{\lambda_{3}^{*} \frac{\partial W}{\partial \lambda_{3}^{*}}-p^{*}}{\lambda_{3}}
$$

is required for the curve fitting and the rock is compressed in the 3 -direction. In general, $\lambda_{1} \neq \lambda_{2}$ and their values are obtained via the relations 


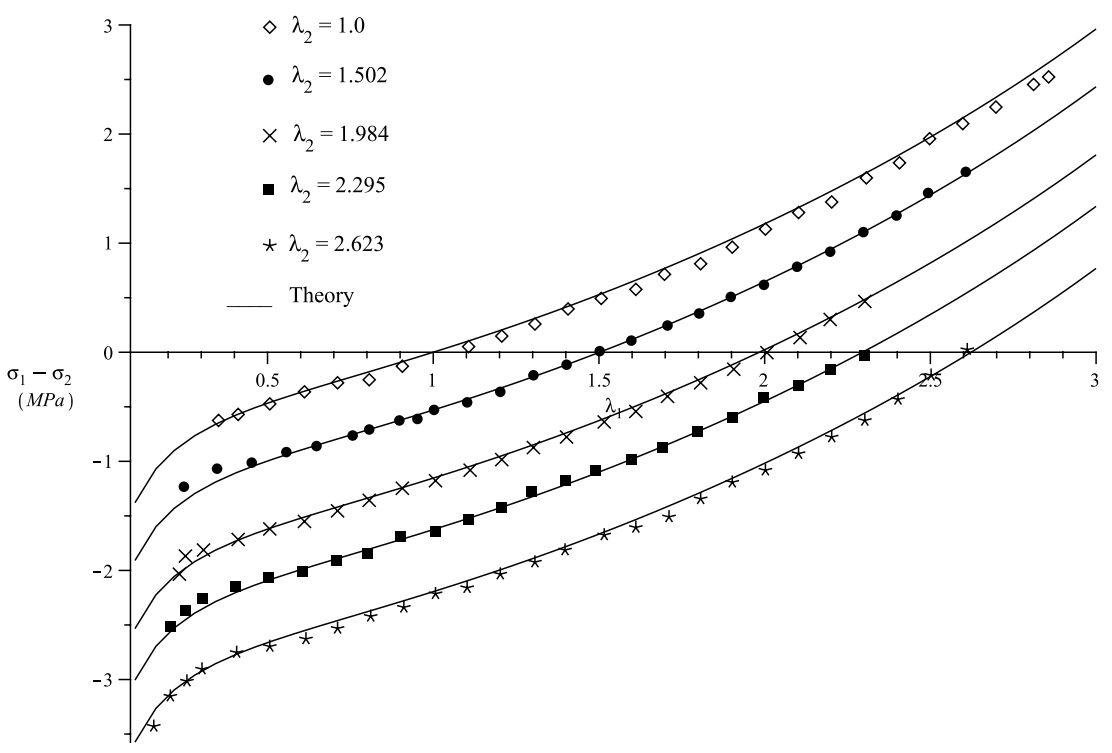

Figure 4. Comparison of theory with the biaxial experimental data of Jones and Treloar ${ }^{50}$.

$$
\lambda_{1}^{*} \frac{\partial W}{\partial \lambda_{1}^{*}}=p^{*}, \quad \lambda_{2}^{*} \frac{\partial W}{\partial \lambda_{2}^{*}}=p^{*}
$$

that correspond to the lateral stress-free condition. To compare our theory with the experiment of Jin et al. ${ }^{51}$, we consider the preferred direction

$$
\boldsymbol{a}=\cos \theta \boldsymbol{g}_{3}+\sin \theta \boldsymbol{g}_{2},
$$

where $\left\{\boldsymbol{g}_{1}, \boldsymbol{g}_{2}, \boldsymbol{g}_{3}\right\}$ is the Cartesian basis and $\theta$ is the angle $\boldsymbol{a}$ makes with the direction $\boldsymbol{g}_{3}$. The simple strain functions

$$
f_{\alpha}(x)=g_{1}(x)=g_{2}(x)=g_{3}(x)=\ln (x), \quad \alpha=1,2, \ldots 7
$$

are used in the curve fitting. The values of the ground-state constants used are

$$
\begin{aligned}
E_{a} & =75.146 \mathrm{GPa}, \quad E_{p}=34.614 \mathrm{GPa} \\
v_{z p} & =0.154, \quad v_{p}=0.374, \quad \mu_{a}=5.48 \mathrm{GPa} .
\end{aligned}
$$

We note that Jin et al. ${ }^{51}$, experimentally, obtained the ground-state constant values:

$$
\begin{aligned}
E_{a} & =16.12 \pm 1.29 \mathrm{GPa}, \quad E_{p}=37.72 \pm 7.04 \mathrm{GPa} \\
v_{z p} & =0.35 \pm 0.15, \quad v_{p}=0.25 \pm 0.01, \quad \mu_{a}=6.87 \pm 1.19 \mathrm{GPa}
\end{aligned}
$$

However, we are not able to satisfactorily fit the data using the data values in (131), but some of our values are quite close to the values obtained by Jin et al. ${ }^{51}$.

We plot the strain-stress equation (126) when $\boldsymbol{a}$ has the directions described by the angles $\theta=0^{\circ}, 30^{\circ}, 45^{\circ}, 60^{\circ}, 90^{\circ}$. The compression curves are given in Fig. 5 and it is clear that our theory capture the behaviour of the experimental data.

To compare with the experimental data given in this section for incompressible materials, we consider a strain energy function of the form ${ }^{14}$

$$
W_{(T)}=\sum_{i=1}^{3}\left[\mu_{T} f_{1}^{2}\left(\lambda_{i}\right)+2\left(\mu_{L}\left(I_{4}\right)-\mu_{T}\right) \zeta_{i} f_{2}^{2}\left(\lambda_{i}\right)\right]+\frac{\beta}{2}\left(I_{4}\right)\left(\sum_{i=1}^{3} \zeta_{i} f_{3}\left(\lambda_{i}\right)\right)^{2},
$$

where the constants $\mu_{T}$ and $\mu_{L}$, represent the elastic shear moduli in the ground state. The other ground state elastic constant $\beta^{41}$ can be related to an elastic constant which has more direct physical interpretations, such as the extension moduli. Since the ground-state constant values when the fibre tension is different from when fibre compression (see Appendix A in Supplementary information), we have,

$$
\mu_{L}\left(I_{4}\right)=l_{p} q_{(p)}\left(I_{4}\right)+l_{n} q_{(n)}\left(I_{4}\right), \quad \beta\left(I_{4}\right)=m_{p} q_{(p)}\left(I_{4}\right)+m_{n} q_{(n)}\left(I_{4}\right), \quad I_{4}=\boldsymbol{a} \cdot \boldsymbol{C a} .
$$

In this section we compare our theory with the uniaxial tension and compression experiment of Chui et al. ${ }^{52}$ and Takaza et al. ${ }^{53}$ multiple angle uniaxial experiment on soft tissue. We note that in Chui et al. ${ }^{52}$ the uniaxial stretch in the fibre direction is the stiffest, where else Takaza et al. ${ }^{53}$ experiment indicates that the transverse stress is 


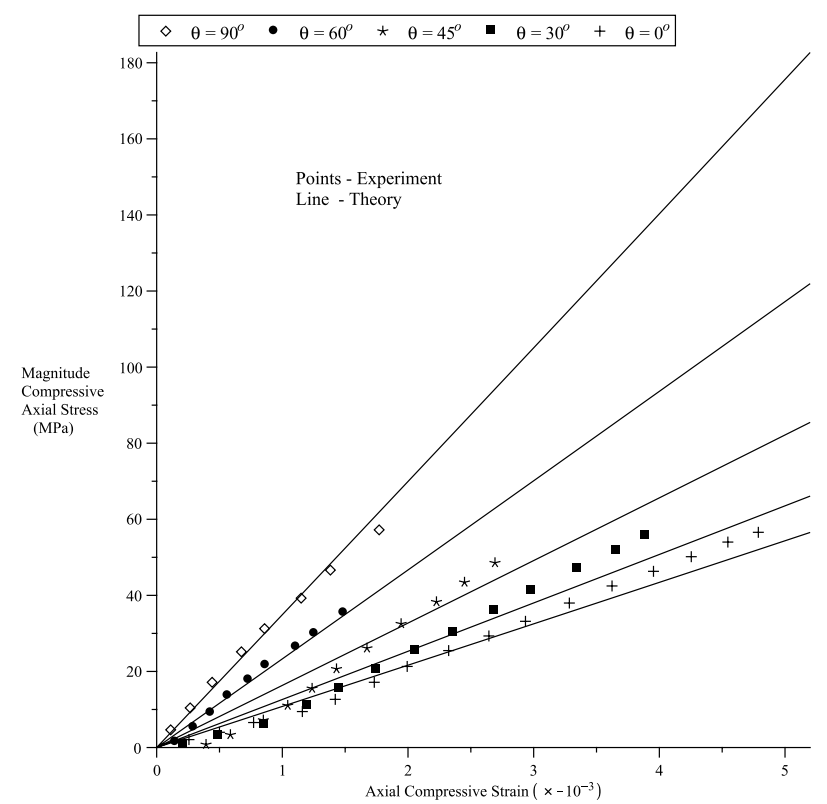

Figure 5. Stress-strain curves for a uniaxial compression deformation. The points are from the experimental test of Jin et al. ${ }^{51}$.

the stiffest. In soft tissues, the initial large extension is generally achieved at relatively low levels of stress with subsequent stiffening at higher levels of extension. This behaviour is due to the recruitment of collagen fibres as they become uncrimped and reach their natural lengths. The inverse error function $\operatorname{erf}^{-1}(x)$ seems a good candidate to describe the above mentioned soft tissue stress-strain behaviour since it has low initial gradients followed by high gradients at higher values of $x$. In view of this, for simplicity, to fit the experiments, we use the strain functions

$$
\begin{aligned}
f_{1}^{2}(x) & =\int_{1}^{x} \frac{4}{\alpha_{1} \sqrt{\pi}} \operatorname{erf}^{-1}\left(\alpha_{1} \ln (y)\right) d y \geq 0, \quad f_{2}^{2}(x)=\int_{1}^{x} \frac{4}{\alpha_{2} \sqrt{\pi}} \operatorname{erf}^{-1}\left(\alpha_{2} \ln (y)\right) d y \geq 0, \\
f_{3}(x) & =\frac{2}{\alpha_{3} \sqrt{\pi}} \operatorname{erf}^{-1}\left(\alpha_{3} \ln (x)\right),
\end{aligned}
$$

where $\alpha_{1-3} \neq 0$ are dimensionless material parameters.

The tensor and vector components used below are with respect to the Cartesian basis $\left\{\boldsymbol{g}_{1}, \boldsymbol{g}_{2}, \boldsymbol{g}_{3}\right\}$. The stressstrain relations are based on that given in (13). We first consider Chui et al. ${ }^{52}$ uniaxial tension and compression experiment. The nominal stresses $T_{f}=\frac{t_{11}}{\lambda_{1}}$ for $\boldsymbol{a} \equiv[1,0,0]^{T}$ and $T_{t}=\frac{t_{11}}{\lambda_{1}}$ for $\boldsymbol{a} \equiv[0,1,0]^{T}$ are plotted. We use $\mu_{T}=200, \beta=0$ and for $\mu_{L}, l_{p}=600$ and $l_{n}=550$. In Fig. 6 we curve fit the data. Using the curve fitted material constant values, in Fig. 7 we predict the experimental data for the transverse stress. It is clear from Fig. 7 that our theory predicts the data quite well.

In the case of Takaza et al..$^{53}$ experiment, we plot the Cauchy stress components $t_{11}$ vs $\lambda_{1}$. In Fig. 8 we curve fit for $\boldsymbol{a} \equiv[1,0,0]^{T}$ (fibre direction stress) and $\boldsymbol{a} \equiv[0,1,0]^{T}$ (transverse direction stress). Using the fitted numerical values for the material constants, we predict the stress for $\boldsymbol{a} \equiv\left[\cos \left(45^{\circ}\right), \sin \left(45^{\circ}\right), 0\right]^{T}($ shown in Fig. 8) and, $\boldsymbol{a} \equiv\left[\cos \left(30^{\circ}\right), \sin \left(30^{\circ}\right), 0\right]^{T}$ and $\boldsymbol{a} \equiv\left[\cos \left(60^{\circ}\right), \sin \left(60^{\circ}\right), 0\right]^{T}$, both shown in Fig. 9 . The values for $\mu_{L}$ are $l_{p}=70$ and $l_{n}=0$. For simplicity, we have assumed, on the onset, $\beta=0$ and $\alpha_{2}=\alpha_{3}$.

Orthotropic. Here, we only consider fitting our theory with the incompressible simple shear experimental data of Dokos et al. ${ }^{54}$ on passive myocardium, where the material can be considered to be orthotropic ${ }^{55}$. We consider the strain energy ${ }^{12}$

$$
\begin{aligned}
W_{(O)}= & \sum_{i=1}^{3}\left[n_{(1)} s^{2}\left(\lambda_{i}\right)+n_{(2)} \zeta_{i} s^{2}\left(\lambda_{i}\right)+n_{(3)} \gamma_{i} s^{2}\left(\lambda_{i}\right)\right]+n_{(5)}\left(\sum_{i=1}^{3} \zeta_{i} s\left(\lambda_{i}\right)\right)^{2} \\
& +n_{(6)}\left(\sum_{i=1}^{3} \gamma_{i} s\left(\lambda_{i}\right)\right)^{2}+n_{(7)} \sum_{i=1}^{3} \zeta_{i} s\left(\lambda_{i}\right) \sum_{i=1}^{3} \gamma_{i} s\left(\lambda_{i}\right)
\end{aligned}
$$

where the strain function 


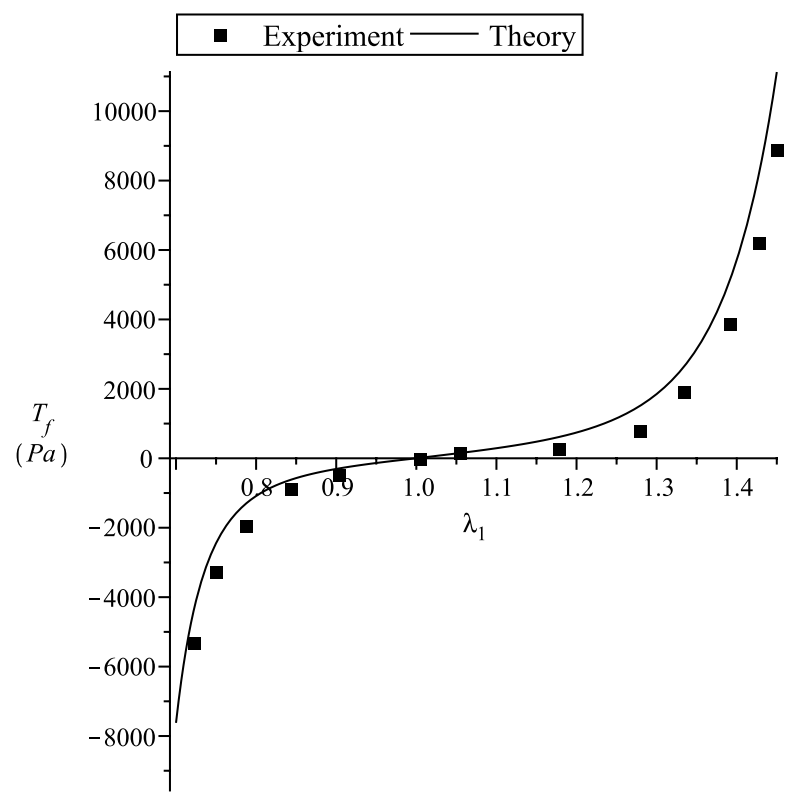

Figure 6. Fitting Chui et al. ${ }^{52}$ porcine liver uniaxial deformation in the fibre direction. $\mu_{T}=200 \mathrm{~Pa}$, $\mu_{L}=600 / 550 \mathrm{~Pa}, \beta=0 \mathrm{~Pa}, \alpha_{1}=\alpha_{2}=\alpha_{3}=5.7$.

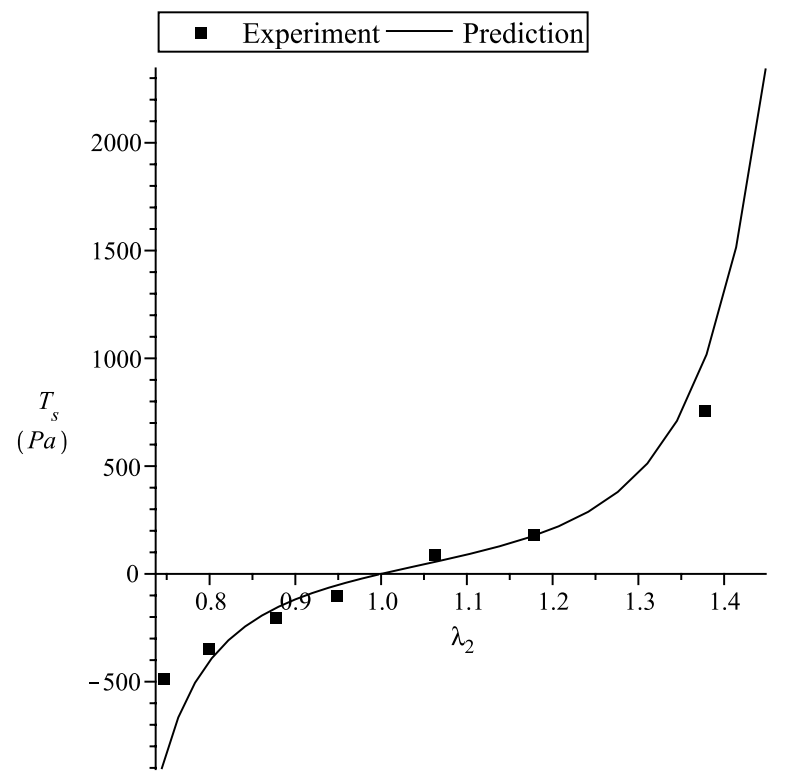

Figure 7. Predicting Chui et al..$^{52}$ porcine liver uniaxial deformation in transverse direction. $\mu_{T}=200 \mathrm{~Pa}$, $\mu_{L}=600 / 550 \mathrm{~Pa}, \beta=0 \mathrm{~Pa}, \alpha_{1}=\alpha_{2}=\alpha_{3}=5.7$.

$$
s(x)=\frac{2}{\rho_{0} \sqrt{\pi}} \operatorname{erf}^{-1}\left(\rho_{0} \ln (x)\right)+\rho_{1}\left(e^{1-x}+x-2\right),
$$

$\operatorname{erf}^{-1}(x)$ is the inverse error function and, $\rho_{o}$ and $\rho_{1}$ are dimensionless material parameters. The tensor and vector components given below are with respect to the Cartesian basis $\left\{\boldsymbol{g}_{1}, \boldsymbol{g}_{2}, \boldsymbol{g}_{3}\right\}$. Following the work of Shariff ${ }^{12}$ the shear stress is given by

$$
\sigma_{12}=2\left[l_{1}\left(\gamma s^{2}+c s\right)+l_{2}\left(\gamma c^{2}-c s\right)+l_{3} \gamma c s\right]
$$

where $\gamma$ is the amount of shear, 


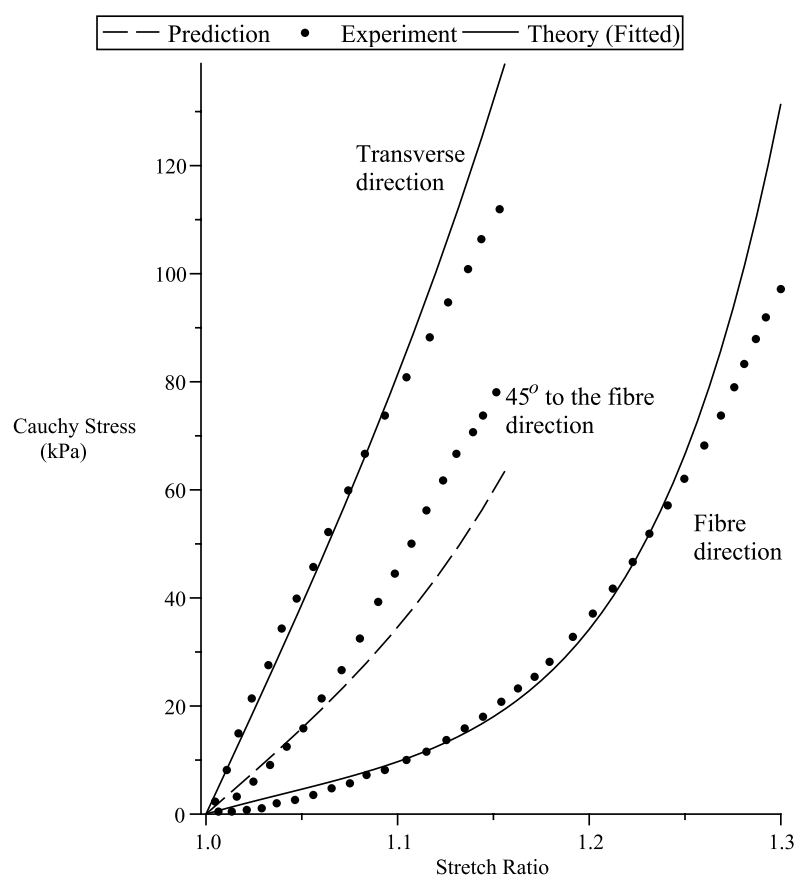

Figure 8. Takaza et al..$^{53}$ uniaxial experiment. $\mu_{T}=200 \mathrm{kPa}, \mu_{L}=70 \mathrm{~Pa}, \beta=0 \mathrm{kPa}, \alpha_{1}=5, \alpha_{2}=\alpha_{3}=3.2$.

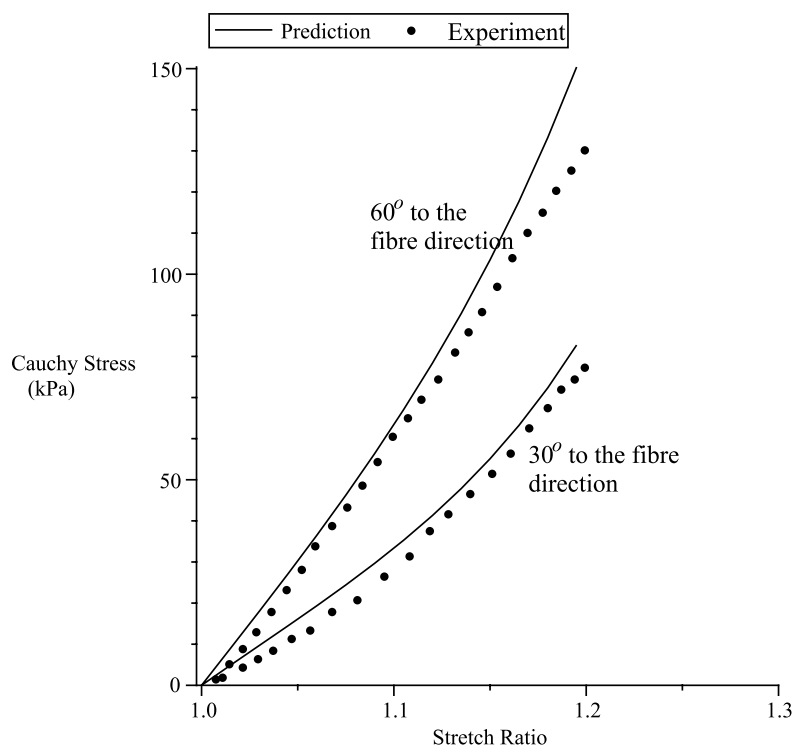

Figure 9. Predicting Takaza et al. ${ }^{53}$ uniaxial experiment. $\mu_{T}=200 \mathrm{kPa}, \mu_{L}=70 \mathrm{kPa}, \beta=0 \mathrm{kPa}$, $\alpha_{1}=5, \alpha_{2}=\alpha_{3}=3.2$.

$$
\begin{gathered}
l_{1}=\frac{1}{2 \lambda_{1}} \frac{\partial W_{(O)}}{\partial \lambda_{1}}, \quad l_{2}=\frac{1}{2 \lambda_{2}} \frac{\partial W_{(O)}}{\partial \lambda_{2}}, \\
l_{3}=\frac{1}{\lambda_{1}^{2}-\lambda_{2}^{2}}\left[\left(\frac{\partial W_{(O)}}{\partial \zeta_{1}}-\frac{\partial W_{(O)}}{\partial \zeta_{2}}\right) a_{1} a_{2}+\left(\frac{\partial W_{(O)}}{\partial \gamma_{1}}-\frac{\partial W_{(O)}}{\partial \gamma_{2}}\right) \iota_{1} \iota_{2}\right], \\
\lambda_{1}=\frac{\gamma+\sqrt{\gamma^{2}+4}}{2} \geq 1, \quad \lambda_{2}=\frac{1}{\lambda_{1}}=\frac{\sqrt{\gamma^{2}+4}-\gamma}{2} \leq 1,
\end{gathered}
$$




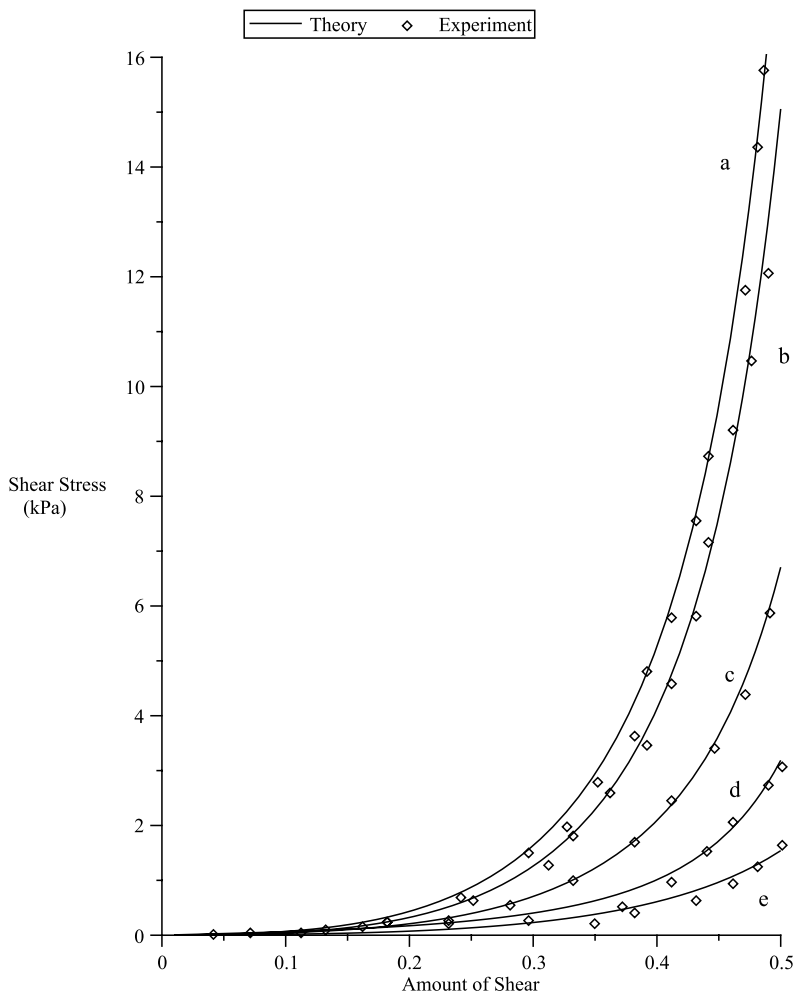

Figure 10. Fitting of our theory with the experiment of Dokos et al. ${ }^{54}$ simple shear data in various sheet/ fibre directions. $\rho_{0}=3.416, \rho_{1}=0.437, n_{(1)}=0.353, n_{(2)}=-0.534, n_{(3)}=0.218, n_{(5)}=47.612$, $n_{(6)}=4.3865, n_{(7)}=-25$..

and

$$
c=\frac{1}{\sqrt{1+\lambda_{1}^{2}}}, \quad s=\frac{\lambda_{1}}{\sqrt{1+\lambda_{1}^{2}}} .
$$

Let $\boldsymbol{a}$ and $\boldsymbol{b}$ represent the fibre and sheet directions ${ }^{55}$, respectively, of the passive myocardium.

In Figure 10, there are six sets of data, however, the experimental data corresponding to the fibre/sheet directions of the passive myocardium with Cartesian components $[1,0,0]^{T} /[0,0,1]^{T}$ and $[0,0,1]^{T} /[1,0,0]^{T}$ are indistinguishable. We note that no experiment is perfect. This indistinguishable behaviour could be caused by minute errors or approximations in the experiment or it could be the actual behaviour of the myocardium specimen or other unknown factors.

We first fit the five sets of data that correspond to fibre/sheet directions with Cartesian components (from top to bottom in Figure 10): (a) $[0,1,0]^{T} /[1,0,0]^{T}(\mathrm{~b})[0,1,0]^{T} /[0,0,1]^{T}(\mathrm{c})[1,0,0]^{T} /[0,1,0]^{T}(\mathrm{~d})[0,0,1]^{T} /[0,1,0]^{T}$ (e) $[1,0,0]^{T} /[0,0,1]^{T}$. It is clear in Figure 10 that very good agreement is indicated between the model and the experimental data.

Using the fitted values, we then predict the set of data that corresponds to the fibre/sheet directions with components $[0,0,1]^{T} /[1,0,0]^{T}$. The predicted curve shown in Fig. 11 is also in good agreement with the experimental data.

\section{Finite element implementation}

In order to obtain numerical solutions for nonlinear isotropic and anisotropic elastic problems, a finite element software, such as Abaqus ${ }^{56}$, requires the end users to supply an explicit expression for the consistent tangent modulus tensor for an invariant-based potential function. In many cases, the consistent (algorithmic) tangent modulus tensor

$$
\mathbb{C}_{\mathbb{T}}=\frac{1}{J} \frac{\partial \boldsymbol{\tau}_{(J)}}{\partial \boldsymbol{D}}
$$

is used in the finite element code, where $\boldsymbol{\tau}_{(J)}$ is the Jaumann rate of the Kirchhoff stress and $\boldsymbol{D}$ is the deformation rate tensor. The consistent tangent modulus tensor requires, among others, the explicit expression for the 4 th-order tensor (see, for example,,$^{57}$ ) 


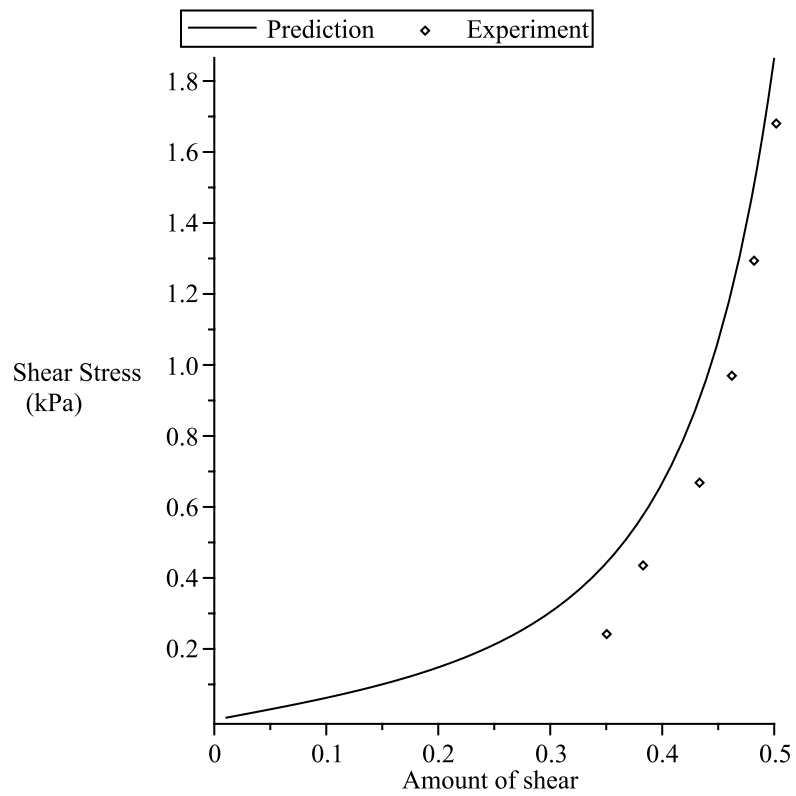

Figure 11. Prediction of fibre/sheet $\left([0,0,1]^{T} /[1,0,0]^{T}\right)$ directions of the simple shear Dokos et al. ${ }^{54}$ experiment. $\rho_{0}=3.416, \rho_{1}=0.437, n_{(1)}=0.353, n_{(2)}=-0.534, n_{(3)}=0.218, n_{(5)}=47.612$, $n_{(6)}=4.3865, n_{(7)}=-25$.

$$
\frac{\partial^{2} W_{(e)}}{\partial \boldsymbol{C} \partial \boldsymbol{C}}
$$

where $W_{(e)}$ represents a strain energy function. Until recently, only consistent tangent modulus tensors for $W_{(e)}$ containing "classical" tensor invariants that can be written explicitly in terms of $\boldsymbol{C}$ were obtained in the literature; this due to the fact that the second derivative of the classical invariants with respect to $C$ (a 4 th order tensor) is easily obtained because they can be expressed explicitly in terms of $\boldsymbol{C}$. However, the consistent tangent modulus tensor for a potential function $W_{(e)}$, containing spectral invariants that cannot be written explicitly in terms of $\boldsymbol{C}$ but can be written explicitly in terms of the eigenvalues and eigenvectors of $\boldsymbol{C}$, has only recently developed by Shariff ${ }^{11}$ and, in view of this, the spectral formulation of the consistent tangent modulus tensor, developed by Shariff ${ }^{11}$, is not so well-known; hence, it may be mistakenly assumed that the spectral consistent tangent modulus tensor cannot be explicitly evaluated and that the proposed model cannot be used in a Finite Element commercial software. So the objective of this section is to report that the spectral consistent tangent modulus tensor can be evaluated using the results given in Shariff ${ }^{11}$.

For isotropic, transversely isotropic and two-preferred direction materials, described above, the strain energy function $W_{(e)}$ contains, invariants of the form

$$
I=\sum_{i=1}^{3}\left(\boldsymbol{u}_{i} \cdot \boldsymbol{G} \boldsymbol{u}_{i}\right) g\left(\lambda_{i}^{*}\right) \text { and } J,
$$

where $\boldsymbol{G}$ is a second order tensor. In Shariff ${ }^{11}$, the tangent modulus tensor (142) for a strain energy function that contains invariants of the form (144) is explicitly formulated. In the case of the strain energy function $W_{(G)}$, given in (103), for a general anisotropic material, the corresponding tangent modulus tensor (142) can be derived using the results given in Shariff ${ }^{10}$; however, due to its complex derivation, we will not derive it here.

\section{Conclusion}

In this contribution, we define a generalized strain function that is similar to the Hill's ${ }^{1}$ strain function and a volumetric function, where they are used to characterize strain energy functions in isotropic or anisotropic elasticity. These strain functions are single variable functions that depend on an invariant with a clear physical meaning, which facilitates the construction of specific forms of the strain energy function, in the sense that a function of a single variable with a clear physical interpretation is easily manageable and this is indicated in "Example of specific forms of $f \alpha$ and $g \alpha$ used in experimental fitting" section; they also facilitate the construction of strain energy functions that are consistent with infinitesimal elasticity as described in sections "Isotropic" to "General anisotropy". The proposed generalized strain functions enable the development of a strain energy function for a general anisotropic material that contains the general 4th order classical stiffness tensor. Having a clear physical interpretation, the spectral invariants are attractive in aiding experiment design. Some previous strain energy functions that appeared in the literature can be considered as special cases of the proposed generalized strain energy functions. The proposed constitutive equations can be easily converted to allow the mechanical influence 
of compressed fibres to be excluded or partial excluded and to model fibre dispersion in collagenous soft tissues, and they can be easily implemented in a Finite Element software. In "Example of specific forms of $f \alpha$ and $g \alpha$ used in experimental fitting" section, we show that the suggested crude specific strain function forms fitted well with experimental data and managed to predict several sets of experimental data. The single-variable-function constitutive equations are expected to set a platform for future modelling of various types of anisotropic elastic solids; future modellers only require to construct specific forms of the functions $f_{\alpha}$ and $g_{\alpha}$ to model their strain energy functions. The extent of the proposed model applicability to different anisotropic needs to be assessed by comparing it with relevant experimental data of a much wider class of anisotropic materials.

Received: 15 August 2021; Accepted: 17 November 2021

Published online: 07 January 2022

\section{References}

1. Hill, R. On constitutive inequalities for simple materials-I. Int. J. Mech. Phys. Solids 16, 229-242 (1968).

2. Ogden, R. W. Large deformation isotropic elasticity: On the correlation of theory and experiment for incompressible rubberlike solids. Proc. R. Soc. Lond. A326, 565-584 (1972).

3. Darijani, H. \& Naghdabadi, R. Constitutive modeling of solids at finite deformation using a second-order stress-strain relation. Int. J. Eng. Sci. 48, 223-236 (2010).

4. Fereidoonnezhad, B., Naghdabadi, R. \& Arghavani, J. A hyperelastic constitutive model for fiber-reinforced rubber-like materials. Int. J. Eng. Sci. 71, 36-44 (2013).

5. Kellermann, D. C. \& Attard, M. M. An invariant-free formulation of neo-Hookean hyperelasticity. Z. für Angew. Math. Mech. 96, 233-252 (2016).

6. O'Shea, D. J., Attard, M. M. \& Kellermann, D. C. Hyperelastic constitutive modelling for transversely isotropic composites and orthotropic biological tissues. Int. J. Solids Struct. 169, 1-20 (2019).

7. O'Shea, D. J., Attard, M. M. \& Kellermann, D. C. Anisotropic hyperelasticity using a fourth-order structural tensor approach. Int. J. Solids Struct. 198, 149-169 (2020).

8. Shariff, M. H. B. M. An anisotropic model for the Mullins effect. J. Eng. Math. 56, 415-435 (2006).

9. Shariff, M. H. B. M. Anisotropic stress-softening model for compressible solids. Z. Angew. Math. Phys. 60, 1112-1134 (2009).

10. Shariff, M. H. B. M. Spectral derivatives in continuum mechanics. Q. J. Mech. Appl. Math. 70(4), 476-479 (2017).

11. Shariff, M. H. B. M. A general spectral nonlinear elastic consistent tangent modulus tensor formula for finite element software. Results Appl. Math. 7, 100113 (2020).

12. Shariff, M. H. B. M. Physical invariant strain energy function for passive myocardium. Biomech. Model. Mechanobiol. 12(2), 215-223 (2013).

13. Shariff, M. H. B. M. Anisotropic separable free energy functions for elastic and non-elastic solids. Acta Mech. 227(11), 3213-3237 (2016).

14. Shariff, M. H. B. M. On the spectral constitutive modelling of transversely isotropic soft tissue: Physical invariants. Int. J. Eng. Sci. 120, 199-219 (2017).

15. Shariff, M. H. B. M. Anisotropic stress softening of residually stressed solids. Proc. R. Soc. A. 477, 20210289. https://doi.org/10. 1098/rspa.2021.0289 (2021).

16. Spencer, A. J. M. Theory of invariants. In Continuum Physics Vol. 1 (ed. Eringen, A. C.) 239-353 (Academic Press, 1971).

17. Shariff, M. H. B. M. \& Merodio, J. Residually stressed two fibre solids: A spectral approach. Int. J. Eng. Sci. 148, 103205 (2020).

18. Crespo, J., Latorre, M. \& Montans, F. J. WYPiWYG hyperelasticity for isotropic, compressible materials. Comput. Mech. 59, 73-92 (2017).

19. Latorre, M. \& Montans, F. J. Extension of the Sussman-Bathe spline-based hyperelastic model to incompressible transversely isotropic materials. Comput. Struct. 122, 13-26 (2013).

20. Latorre, M. \& Montans, F. J. What-You-Prescribe-Is-What-You-Get orthotropic hyperelasticity. Comput. Mech. 53(6), 1279-1298 (2014).

21. Latorre, M. \& Montans, F. J. WYPiWYG hyperelasticity without inversion formula: Application to passive ventricular myocardium. Comput. Struct. 185, 47-58 (2017).

22. Rosa, E. D., Latorre, M. \& Montáns, F. J. Capturing anisotropic constitutive models with WYPiWYG hyperelasticity; and on consistency with the infinitesimal theory at all deformation levels. Int. J. Non-Linear Mech. 96, 75-92 (2017).

23. Shariff, M. H. B. M. Direction dependent orthotropic model for Mullins materials. Int. J. Solids Struct. 51, 4357-4372 (2014).

24. Mielke, A. \& Tomas, R. Rate-independent damage processes in nonlinear elasticity. Math. Models Methods Appl. Sci. 16(2), 177-209 (2006).

25. Misra, A. \& Payam, P. Granular micromechanics model for damage and plasticity of cementitious materials based upon thermomechanics. Math. Mech. Solids 25(10), 1778-1803 (2020).

26. Placidi, L., Barchiesi, E., Misra, A. \& Timofeev, D. Micromechanics-based elasto-plastic-damage energy formulation for strain gradient solids with granular microstructure. Continu. Mech. Thermodyn. 66, 1-29 (2021).

27. Barchiesi, E., Misra, A., Placidi, L. \& Turco, E. Granular micromechanics-based identification of isotropic strain gradient parameters for elastic geometrically nonlinear deformations. Zeitschrift für Angewandte Mathematik und Mechanik, e202100059 (2021).

28. Soldatos, K. P., Shariff, M. H. B. M. \& Merodio, J. On the constitution of polar fibre reinforced materials. Mech. Adv. Meter. Struct.https://doi.org/10.1080/15376494.2020.1729449 (2020).

29. Shariff, M. H. B. M. Nonlinear orthotropic elasticity: Only six invariants are independent. J. Elast. 110, 237-241 (2013).

30. Shariff, M. H. B. M. \& Bustamante, R. On the independence of strain invariants of two preferred direction nonlinear elasticity. Int. J. Eng. Sci. 97, 18-25 (2015).

31. Shariff, M. H. B. M. The number of independent invariants of an n-preferred direction anisotropic solid. Math. Mech. Solids 22(10), 1989-1996 (2017).

32. Shariff, M. H. B. M. On the number of independent invariants for $\mathrm{m}$ unit vectors and $\mathrm{n}$ symmetric second order tensors. Eng. Lett. 29(2), 509-515 (2021).

33. Destrade, M. Mac., Donald, B., Murphy, J. \& Saccomandi, G. At least three invariants are necessary to model the mechanical response of incompressible, transversely isotropic materials. Comput. Mech. 52(4), 959-969 (2013).

34. Horgan, C. O. \& Smayda, M. G. The importance of the second strain invariant in the constitutive modeling of elastomers and soft biomaterials. Mech. Mater. 51, 43-52 (2012).

35. Murphy, J. \& Biwa, S. The counterintuitive mechanical response in simple tension of arterial models that are separable functions of the I1, I4, I6 invariants. Int. J. Non-Linear Mech. 90, 72-81 (2017).

36. Valanis, K. C. \& Landel, R. F. The strain-energy function of hyperelastic material in terms of the extension ratios. J. Appl. Phys. 38, 2997-3002 (1967). 
37. Shariff, M. H. B. M. Strain energy function for filled and unfilled rubberlike material. Rubber Chem. Technol. 73, 1-21 (2000).

38. Shariff, M. H. B. M. \& Parker, D. F. An extension of Key's principle to nonlinear elasticity. J. Eng. Math. 37, 171-190 (2000).

39. Ogden, R. W. Non-linear Elastic Deformations (Ellis Horwood, 1984).

40. Hencky, H. Uber die Form des Elastizitatsgesetzes bei ideal elastischen Stoffen. Z. Techn. Phys. 9, 215-220 (1928).

41. Spencer, A. J. M. Constitutive Theory of the Mechanics of Fiber Reinforced Composites, CISM Courses and Lectures No. 282 (Springer, 1984).

42. Shariff, M. H. B. M. \& Bustamante, R. A. spectral approach for nonlinear transversely isotropic elastic bodies, for a new class of constitutive equation: Applications to rock mechanics. Acta Mech. 231(11), 4803-4818 (2020).

43. Spencer, A. J. M. Continuum Mechanics (Dover, 1980).

44. Betten, J. Invariants of fourth-order tensors. In Applications of Tensor Functions in Solid Mechanics, CISM Courses and Lectures No. 292 (ed. Boehler, J. P.) 171-186 (Wien, 1987)

45. Marckmann, G. \& Verron, E. Comparison of hyperelastic models for rubber-like materials. Rubber Chem. Technol. 79(5), 835-858. https://doi.org/10.5254/1.3547969 (2006).

46. Ogden, R. W., Saccomandi, G. \& Sgura, I. Fitting hyperelastic models to experimental data. Comput. Mech. 34(6), 484-502 (2004).

47. Steinmann, P., Mokarram Hossain, M. \& Possart, G. Hyperelastic models for rubber-like materials: consistent tangent operators and suitability for Treloar's data. Arch. Appl. Mech. 82(9), 1183-1217. https://doi.org/10.1007/s00419-012-0610-z (2012).

48. Blatz, P. J. \& Ko, W. L. Application of finite elastic theory to the deformation of rubbery materials. Trans. Soc. Rheol. VI, 223-251 (1962).

49. Treloar, L. R. G. Stress-strain data for vulcanised rubber under various types of deformation. Trans. Faraday Soc. 40, 59-70 (1940).

50. Jones, D. F. \& Treloar, L. R. G. The properties of rubber in pure homogeneous strain. J. Phys. D Appl. Phys. 8(11), 1285-1304 (1975).

51. Jin, W., Lia, Z., Jin, W., Hambletona, C. \& Cusatisa, G. Anisotropic elastic, strength, and fracture properties of Marcellus shale. Int. J. Rock Mech. Min. Sci. 109, 124-137 (2018).

52. Chui, C., Kobayashi, E., Chen, X., Hisada, T. \& Sakuma, I. Transversely isotropic properties of porcine liver tissue: Experiments and constitutive modeling. Med. Biol. Eng. Comput. 45, 99-106 (2007).

53. Takaza, M., Moerman, K. M., Gindre, J., Lyons, G. \& Simms, C. K. The anisotropic mechanical behaviour of passive skeletal muscle tissue subjected to large tensile strain. J. Mech. Behav. Biomed. Mater. 17, 209-220 (2013).

54. Dokos, S., Smaill, B. H., Young, A. A. \& LeGrice, I. J. Shear properties of passive ventricular myocardium. Am. J. Physiol. Heart Circ. Physiol. 283, H2650-H2659 (2002).

55. Holzapfel, G. A. \& Ogden, R. W. Constitutive modeling of passive myocardium: A structurally based framework of material characterization. Philos. Trans. R. Soc. A 367, 3445-3475 (2009).

56. Abaqus. Theory Manual (Dassault Systemes Simulia, 2013).

57. Rodriguez, J. \& Merodio, J. Helical buckling and postbuckling of pre-stressed cylindrical tubes under finite torsion. Finite Elem. Anal. Design 112, 1-10 (2016).

\section{Author contributions}

The single author M.H.B.M.S. wrote the whole manuscript.

\section{Competing interests}

The author declares no competing interests.

\section{Additional information}

Supplementary Information The online version contains supplementary material available at https://doi.org/ 10.1038/s41598-021-03842-3.

Correspondence and requests for materials should be addressed to M.H.B.M.S.

Reprints and permissions information is available at www.nature.com/reprints.

Publisher's note Springer Nature remains neutral with regard to jurisdictional claims in published maps and institutional affiliations.

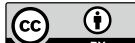

Open Access This article is licensed under a Creative Commons Attribution 4.0 International License, which permits use, sharing, adaptation, distribution and reproduction in any medium or format, as long as you give appropriate credit to the original author(s) and the source, provide a link to the Creative Commons licence, and indicate if changes were made. The images or other third party material in this article are included in the article's Creative Commons licence, unless indicated otherwise in a credit line to the material. If material is not included in the article's Creative Commons licence and your intended use is not permitted by statutory regulation or exceeds the permitted use, you will need to obtain permission directly from the copyright holder. To view a copy of this licence, visit http://creativecommons.org/licenses/by/4.0/.

(c) The Author(s) 2022 